Submitted to the Annals of Probability

arXiv: arXiv: 1711.01258

\title{
ON THE TRANSIENT (T) CONDITION FOR RANDOM WALK IN MIXING ENVIRONMENT
}

\author{
By Enrique Guerra Aguilar* \\ Pontificia Universidad Católica de Chile
}

\begin{abstract}
We prove a ballistic strong law of large numbers and an invariance principle for random walks in strong mixing environments, under condition $(T)$ of Sznitman (cf. [Sz01]). This weakens for the first time Kalikow's ballisticity assumption on mixing environments and proves the existence of arbitrary finite order moments for the approximate regeneration time of $\mathrm{F}$. Comets and O. Zeitouni [CZ02]. The main technical tool in the proof is the introduction of renormalization schemes, which had only been considered for i.i.d. environments.
\end{abstract}

1. Introduction. Random walk in a random environment (RWRE) is a well-known stochastic model for random motion in random media, which presents a wide range of applications going from DNA replication models [Ch62] up to for instance, a prototype for the study of turbulent behavior in fluids [Si82]. The model describes the stochastic evolution of a particle on the lattice $\mathbb{Z}^{d}$, where its transition probabilities are in turn random. Within this framework, it is a fundamental and challenging question to find the minimal local assumption that provides a given asymptotic behaviour for the walk. For technical issues, the local assumption is usually strengthened to an assumption of ballistic-type, the target therefore is to prove a given behavior from one condition on the environment and one ballisticity condition. In this work, assuming a mixing condition on the environment and condition $(T)$ of Sznitman (cf. [Sz01]-[Sz02]), we shall prove ballistic regime complemented with a diffusive scaling limit for the walk.

In the one-dimensional setting one can find almost complete descriptions about RWRE asymptotic laws, scaling limits and connections between different large scale concepts (see [Ze04], Chapter 2 for a comprehensive review for $d=1$ ). Throughout this article we focus on the higher dimensional case, i.e. when the underlying dimension $d$ of the walk is greater than 1. A key role

* Partially Supported by CAPES PNPD20130824, CONICYT FONDECYT Postdoctorado 3180255 and Nucleus Millenium Stochastic Models of Complex and Disordered Systems NC130062

MSC 2010 subject classifications: Primary 60K37; secondary 82D30

Keywords and phrases: Random walk in random environment, Ballisticity Conditions, Strong mixing environments 
to prove our results will be played by renormalization methods for mixing environments. The strategy of renormalization for RWRE was introduced by Alain-Sol Sznitman in [Sz00], and further developments can be found in subsequent articles as [Sz01], [Sz02] and [BDR14], among others. In this article renormalization for RWRE is related to the theoretical construction of strategies that allow the walker to escape from traps (typically we are concerned with traps which are slabs or large boxes) by the appropriate boundary side, with high probability. Overall, the construction of these strategies involves the use of smaller traps to be considered therein, which turns out a recursive procedure of renormalization nature. For i.i.d. random environments, estimates for exit probabilities from traps are established with the help of the renewal structure of A-S. Sznitman and M. Zerner [SZ99], a higher dimensional analogue of the one previously introduced by H. Kesten in [Ke77] for one-dimensional RWRE.

On the other hand, a kind of renewal structure for mixing environments was introduced by F. Comets and O. Zeitouni in [CZ01]. This is an approximate renewal structure for general mixing random environments. Indeed, the authors studied a quit weak mixing assumption, the so-called cone mixing condition. They proved a law of large numbers for a class of strong ballistic RWRE, where the hypotheses are: a strengthened form of Kalikow's condition (cf. (6.1)), integrability conditions for the approximate regeneration time and the cone mixing assumption on the environment (cf. [CZ01], Theorem 3.4). As the present work shows, the integrability conditions can be disposed provided we assume stronger mixing conditions on the environment. Alongside, a stronger mixing condition on the environment has been investigated by F. Rassoul-Agha [RA03], which appears in the context of spin-glass systems at high temperature and was introduced by R. Dobrushin and S. Shlosman (cf. [DS85], see also [Ma99] as a further reference). Under Kalikow's condition, F. Rassoul-Agha proved a ballistic strong law of large numbers by virtue of an appropriate extension of Kozlov's theorem (see $[$ Ko85]). The approach to prove such extension appears when one sees the stochastic evolution of the system from the point of view of the particle. As a matter of fact, Rassoul-Agha's proof does not need to assume a stronger version of Kalikow's condition, as was done in the aforementioned result of [CZ01]. However the point of view of the particle relies on ergodic matters, making hard to visualize a proof for the central limit theorem from this technique.

In this article we shall see that assuming condition $(T)$ along with renormalization type of arguments, one has a Brownian scaling limit under the natural scaling of a ballistic walk. Indeed, we shall reconstruct or give mean- 
ing to part of Sznitman's work [Sz00]-[Sz01]-[Sz03] for i.i.d. environments, in a mixing setting. Thus the present article is fully connected with the spirit of Feynman's phrase: "There is pleasure in recognising old things from a new viewpoint". As a result of that recognition we will be able to weaken the ballisticity assumption from Kalikow's to Sznitman's $(T)$ condition, proving ballistic behaviour and a central limit theorem. Remarkably, we obtain analogously to the i.i.d. case the spirit of a RWRE result: ballistic behaviour from one environment and one ballisticity assumptions. We also open a path for the investigation of ballistic behaviour under weaker assumptions than Kalikow's condition, and we provide a partial answer to an open problem formulated in [CZ01] about the meaning of Sznitman transient conditions in a mixing setting (cf. [CZ01], pp. 912-913, 6. Concluding remarks, item 3).

It is convenient at this point to fix some notation. We only consider what is called in the RWRE literature as a uniform elliptic random environment, which means that the walk has strictly uniform positive jump probabilities to each nearest neighbour sites. More precisely, we pick an integer $d>1$ along with a positive real number $\kappa \in(0,1 /(4 d)]$ and denote by $\mathcal{P}_{\kappa}$ the $2 d$-dimensional simplex:

$$
\mathcal{P}_{\kappa}:=\left\{z \in \mathbb{R}^{2 d}: \Sigma_{1 \leq i \leq 2 d} z_{i}=1, z_{i} \geq 2 \kappa \forall i \in[1,2 d]\right\} .
$$

We consider the product space $\Omega=\left(\mathcal{P}_{\kappa}\right)^{\mathbb{Z}^{d}}$ which is tacitly endowed with its canonical product $\sigma$-algebra denoted by $\mathfrak{F}_{\Omega}$ and, for the time being, fix a probability law $\mathbb{P}$ on $\mathfrak{F}_{\Omega}$. Next, for a given random element $\omega:=$ $(\omega(y, e))_{\left\{y \in \mathbb{Z}^{d}, e \in \mathbb{Z}^{d}:|e|=1\right\}} \in \Omega$, and $x \in \mathbb{Z}^{d}$, we define the quenched law $P_{x, \omega}$ as the law of the canonical Markov chain $\left(X_{n}\right)_{n \geq 0}$ with state space $\mathbb{Z}^{d}$ and stationary transition probabilities satisfying

$$
\begin{aligned}
& P_{x, \omega}\left[X_{0}=x\right]=1, \\
& P_{x, \omega}\left[X_{n+1}=X_{n}+e \mid X_{n}\right]=\omega\left(X_{n}, e\right),|e|=1 .
\end{aligned}
$$

One then defines the annealed law $P_{x}$ of the random walk via the semidirect product $\mathbb{P} \otimes P_{x, \omega}$ on the product $\sigma$-algebra of the space $\Omega \times\left(\mathbb{Z}^{d}\right)^{\mathbb{N}}$. It will be convenient to denote by $|\cdot|_{1},|\cdot|_{2}$ and $|\cdot|_{\infty}$, the $\ell_{1}, \ell_{2}$ and $\ell_{\infty}$ norms, respectively. Furthermore, in this article we will deal with distances between sets, and for instance for $A, B \subset \mathbb{Z}^{d}$, the symbol $d_{1}(A, B)$ stands for the $\ell_{1}$ distance between sets $A$ and $B$, i.e. $d_{1}(A, B):=\inf \left\{|x-y|_{1}, x \in A, y \in B\right\}$. Following X. Guo in [Gu14], we now introduce the type of randomness on the environment of interest for us. For this end, let us first recall the definition of $r$-Markovian field. 
Definition 1.1. For $r>1$, let $\partial^{r} V=\left\{z \in \mathbb{Z} \backslash V: \exists y \in V,|z-y|_{1} \leq r\right\}$ be the $r$-boundary of the set $V \subset \mathbb{Z}$. A random environment $\left(\mathbb{P}, \mathfrak{F}_{\Omega}\right)$ on $\mathbb{Z}^{d}$ is called $r$-Markovian if for any finite $V \subset \mathbb{Z}^{d}$,

$$
\mathbb{P}\left[\left(\omega_{x}\right)_{x \in V} \in \cdot \mid \mathfrak{F}_{V^{c}}\right]=\mathbb{P}\left[\left(\omega_{x}\right)_{x \in V} \in \cdot \mid \mathfrak{F}_{\partial^{r} V}\right], \mathbb{P}-\text { a.s. }
$$

where $\mathfrak{F}_{\Lambda}=\sigma\left(\omega_{x}, x \in \Lambda\right)$.

Let $C$ and $g$ be positive real numbers. We will say that an $r$-Markovian field $\left(\mathbb{P}, \mathfrak{F}_{\Omega}\right)$ satisfies strong mixing condition $(\mathbf{S M})_{C, g}$ if for all finite subsets $\Delta \subset V \subset \mathbb{Z}^{d}$ with $d_{1}\left(\Delta, V^{c}\right) \geq r$, and $A \subset V^{c}$,

$$
\frac{d \mathbb{P}\left[\left(\omega_{x}\right)_{x \in \Delta} \in \cdot \mid \eta\right]}{d \mathbb{P}\left[\left(\omega_{x}\right)_{x \in \Delta} \in \cdot \mid \eta^{\prime}\right]} \leq \exp \left(C \sum_{x \in \partial^{r} \Delta, y \in \partial^{r} A} e^{-g|x-y|_{1}}\right)
$$

for $\mathbb{P}$-almost all pairs of configurations $\eta, \eta^{\prime} \in \Omega$ which agree over the set $V^{c} \backslash A$. Here we have used the notation

$$
\mathbb{P}\left[\left(\omega_{x}\right)_{x \in \Delta} \in \cdot \mid \eta\right]=\left.\mathbb{P}\left[\left(\omega_{x}\right)_{x \in \Delta} \in \cdot \mid \mathfrak{F}_{V^{c}}\right]\right|_{\left(\omega_{x}\right)_{x \in V^{c}=\eta}}
$$

We will also need a condition which is somehow weaker than the previous one. We say an $r$-Markovian field $\left(\mathbb{P}, \mathfrak{F}_{\Omega}\right)$ satisfies Guo's strong mixing condition $(\mathbf{S M G})_{C, g}$ if for all finite subsets $\Delta \subset V \subset \mathbb{Z}^{d}$ with $d_{1}\left(\Delta, V^{c}\right) \geq r$, and $A \subset V^{c}$,

$$
\frac{d \mathbb{P}\left[\left(\omega_{x}\right)_{x \in \Delta} \in \cdot \mid \eta\right]}{d \mathbb{P}\left[\left(\omega_{x}\right)_{x \in \Delta} \in \cdot \mid \eta^{\prime}\right]} \leq \exp \left(C \sum_{x \in \Delta, y \in A} e^{-g|x-y|_{1}}\right)
$$

with the same notation as above.

Throughout this article, condition $(\mathbf{S M})_{C, g}$ will be the main assumption on the environment and we will use condition (SMG) $C, g$ only with the purpose of using an asymptotic more general assumption. Strictly speaking, $(\mathbf{S M G})_{C, g}$ is not implied by condition $(\mathbf{S M})_{C, g}$, but in asymptotic terms it is harder to work with $(\mathbf{S M G})_{C, g}$. The so-called Dobrushin-Sloshman condition implies $(\mathbf{S M})_{C, g}$, for some constants $C$ and $g$ (cf. Lemma 9 of [RA03]). We will not define Dobrushin-Sloshman condition and we refer to [DS85] for the original reference about this mixing assumption, and also to [RA03] for a discussion more suitable for our purposes.

We will now introduce condition $(\mathbf{T})_{\ell}$, where $\ell$ is an element of the $d$ dimensional unit sphere $\mathbb{S}^{d-1}$ (cf. [Sz01]-[Sz02]). As a result of Lemma 2.2, 
for $\ell \in \mathbb{S}^{d-1}$ we can and do say that condition $(\mathbf{T})_{l}$ is satisfied, if there exists a neighborhood $U \subset \mathbb{S}^{d-1}$ of $\ell$, so that for some $b, \tilde{b}>0$ one has that

$$
\limsup _{L \rightarrow \infty} L^{-1} \log \left(P_{0}\left[\widetilde{T}_{-b L}^{l^{\prime}}<T_{\tilde{b} L}^{l^{\prime}}\right]\right)<0
$$

holds, for all $l^{\prime} \in U$, where we have used the standard notation: if $a \in \mathbb{R}$ and $u \in \mathbb{R} \backslash\{0\}, T_{a}^{u}$ and $\widetilde{T}_{a}^{u}$ denote stopping times defined as:

$$
T_{a}^{u}:=\inf \left\{n \geq 0: X_{n} \cdot u \geq a\right\} \text { and } \widetilde{T}_{a}^{u}:=T_{-a}^{-u} .
$$

We will point out that the exponential moment version of this condition (which is the original definition of [Sz01], page 726) does not make sense since we do not have planar regeneration times in mixing. Rather, we have approximate cone regeneration times (cf. Section 2). The exponential moment and slab definitions are equivalent for i.i.d environments (cf. [Sz02], Theorem 1.1).

Our main result rests on a further assumption.

DEFINITION 1.2. We say that assumption $(\boldsymbol{R})_{g, \kappa}$ is satisfied if:

$$
g>18 \log \left(\frac{1}{\kappa}\right) .
$$

For i.i.d. environments one can take $g$ arbitrarily large in either: (1.2) or (1.3). On the other hand, one can construct non-degenerate $r$-Markovian fields with properties (1.2) or (1.3) for any given intensity parameter $g>0$ (cf. [D094]-[DS85]-[Ma99]).

We obtain an annealed functional central limit for the natural scaling of a ballistic walk under the a priori transient $(\mathbf{T})_{\ell}$ condition.

TheOREm 1.3. Let $C, g>0$ and $\ell \in \mathbb{S}^{d-1}$. Suppose that the $R W R E$ satisfies conditions $(\boldsymbol{T})_{\ell}$, either: $(\boldsymbol{S M})_{C, g}$ or $(\boldsymbol{S} \boldsymbol{M G})_{C, g}$ and (1.5). Then there exist a deterministic non-degenerate covariance matrix $R$ and a deterministic vector $v$ with $v \cdot \ell>0$, such that under $P_{0}$; with

$$
S_{n}(t):=\frac{X_{[n t]}-v t}{\sqrt{n}},
$$

the path $S_{n}(t)$ taking values in the space of right continuous functions possessing left limits equipped with the supremum norm, converges in law to a standard Brownian motion with covariance matrix $R$.

imsart-aop ver. 2014/10/16 file: trenormrev.tex date: November 19, 2018 
It is not our subject finite dependent environments, how- ever let us mention that we can avoid the use of assumption (1.5) in that case. We refer to Remark 4.6 for a sketch of proof.

Theorem 1.3 is the first result in the direction of weakening Kalikow's condition for a class of ballistic random walks in mixing environments. It is also for mixing environments the first time that an invariance principle is established from only one ballisticity condition. Denoting Kalikow's condition in direction $\ell \in \mathbb{S}^{d-1}$ by $(\mathbf{K})_{\ell}$ (cf. (6.1)), we will prove in Section 6 the implication: $(\mathbf{K})_{\ell} \rightarrow(\mathbf{T})_{\ell}$. In general, the converse implication fails and we refer to Section 6 for further details.

We will now describe in some detail the contents and structure of this article. Section 2 gives equivalent formulations for condition (T) and introduces the asymptotic renewal structure of Comets and Zeitouni [CZ01]. The random variable $\tau_{1}$ introduced there produces an almost regeneration property. The term almost is made precise in Section 3, Proposition 3.1 and Corollary 3.3. The crucial Section 4 is mostly concerned with Proposition 4.1 and 4.5. These propositions show finiteness of some exponential moments for the random variable $\left|X_{\tau_{1}}\right|_{2}$ and a stretched exponential control on the probability of large fluctuation along the orthogonal space to the approximate asymptotic direction. Section 5 proves Theorem 1.3 using the stretched exponential controls of Proposition 4.5 together with renormalization to bound the tails of $\tau_{1}$. The last section will be devoted to prove that Kalikow's condition is stronger than $(\mathbf{T})$. We shall also see under Kalikow's condition that a strong law of large numbers of ballistic nature holds without the use of assumption (1.5), recovering by others methods F. Rassoul-Agha's theorem [RA03] under a slightly weaker mixing hypothesis. Nevertheless, since the main assumption to construct the invariant measure $\widehat{\mathbb{P}}_{\infty} \ll \mathbb{P}$ in [RA03] appeals to a ballistic estimate which is provided by Kalikow's condition (cf. (6.19)) and the mixing condition is comparable to ours (cf. Lemma 7 in [RA03]), it is possible that Rassoul-Agha's approach would apply under our assumptions as well.

2. The Transient $(T)$ Condition and The Approximate Renewal Structure.. We shall introduce the condition $(T)$ and recall the approximate regeneration time introduced in [CZ01] by F. Comets and O. Zeitouni.

2.1. On the $(T)$ Condition. We begin with recalling the definition of directed system of slabs as in [Sz02].

Definition 2.1. We say that $l_{0}, l_{1}, \ldots, l_{k} \in \mathbb{S}^{d-1}, a_{0}=1, a_{1}, \ldots, a_{k}>$ $0, b_{0}, \ldots, b_{k}>0$ generate an $l_{0}$-directed systems of slabs of order 1 , when

imsart-aop ver. 2014/10/16 file: trenormrev.tex date: November 19, 2018 
- $l_{0}, l_{1} \ldots, l_{k}$ generate $\mathbb{R}^{d}$

- $\mathcal{D}=\left\{x \in \mathbb{R}^{d}: x \cdot l_{0} \in\left[-b_{0}, 1\right], l_{i} \cdot x \geq-b_{i}, i \in[1, k]\right\} \subset\left\{x \in \mathbb{R}^{d}\right.$ : $\left.l_{i} \cdot x<a_{i}, \forall i \in[1, k]\right\}$

- $\lim \sup _{M \rightarrow \infty} M^{-1} \log P_{0}\left[\widetilde{T}_{-b_{i} M}^{l_{i}}<T_{a_{i} M}^{l_{i}}\right]<0$, for $i \in[0, k]$, with the convention $a_{0}=1$.

For positives real numbers $L, L^{\prime}$ and $l \in \mathbb{S}^{d-1}$, we introduce the box $B_{L, L^{\prime}, l}(x)$ as

$$
B_{L, L^{\prime}, l}(x):=x+R\left((-L, L) \times\left(-L^{\prime}, L^{\prime}\right)^{d-1}\right) \cap \mathbb{Z}^{d},
$$

where $R$ is a rotation of $\mathbb{R}^{d}$ with $R\left(e_{1}\right)=l$ (the specific form of such a rotation is immaterial for our purposes) and $x \in \mathbb{Z}^{d}$. For $V \subset \mathbb{Z}^{d}$, we set $\partial V=\partial^{1} V$. Then for a given box $B_{L, L^{\prime}, l}(x)$ we define its positive boundary $\partial^{+} B_{L, L^{\prime}, l}(x)$ by

$$
\partial^{+} B_{L, L^{\prime}, l}(x):=\partial B_{L, L^{\prime}, l}(x) \cap\left\{y \in \mathbb{Z}^{d}:(y-x) \cdot l \geq L\right\} .
$$

We also introduce for $A \subset \mathbb{Z}^{d}$ the exit time $T_{A}$ and the entrance time $H_{A}$ via:

$$
\begin{aligned}
& T_{A}:=\inf \left\{n \geq 0: X_{n} \notin A\right\} \text { and } \\
& H_{A}:=\inf \left\{n \geq 0: X_{n} \in A\right\} .
\end{aligned}
$$

We can then prove

LEMMA 2.2. The following assertions are equivalents:

(i) There exist data $l_{0}, l_{1}, \ldots, l_{k} \in \mathbb{S}^{d-1}, a_{0}=1, a_{1}, a_{2}, \ldots, a_{k}>0$, $b_{0}, b_{1}, \ldots, b_{k}>0$ generating an $l_{0}$-directed systems of slabs of order 1 .

(ii) For some positive constants $b$ and $\hat{r}$, and large $M$, there are finite subsets $\Delta_{M} \subset \mathbb{Z}^{d}$, with $0 \in \Delta_{M} \subset\left\{x \in \mathbb{Z}^{d}: x \cdot l_{0} \geq-b M\right\} \cap\left\{x \in \mathbb{R}^{d}\right.$ : $\left.|x|_{2} \leq \hat{r} M\right\}$ and

$$
\limsup _{M \rightarrow \infty} M^{-1} \log P_{0}\left[X_{T_{\Delta_{M}}} \notin \partial^{+} \Delta_{M}\right]<0,
$$

where $\partial^{+} \Delta_{M}=\partial \Delta \cap\left\{x \in \mathbb{R}^{d}: x \cdot l_{0} \geq M\right\}$.

(iii) For some $\mathfrak{r}>0$, one has

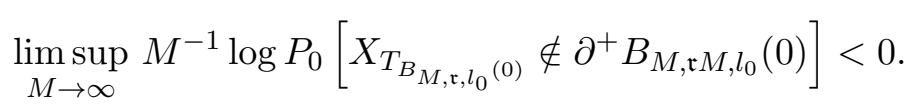

imsart-aop ver. 2014/10/16 file: trenormrev.tex date: November 19, 2018 
Furthermore, in case of any of them holds, we say that $(\boldsymbol{T})_{l_{0}}$ (to be read as condition $T$ in direction $l_{0}$ ) holds.

Proof. The proof of (i) $\Rightarrow$ (ii) can be found in [Sz02], pp 516-517. Therefore, we turn to prove (ii) $\Rightarrow$ (iii). By (ii), there exist $b, \hat{r}>0$, so that for large $M$ there are finite subsets $\Delta_{M}$ with $0 \in \Delta_{M} \subset\left\{x \in \mathbb{Z}^{d}: x \cdot l_{0} \geq\right.$ $-b M\} \cap\left\{x \in \mathbb{R}^{d}:|x|_{2} \leq \hat{r} M\right\}$ and

$$
\limsup _{M \rightarrow \infty} M^{-1} \log P_{0}\left[X_{T_{\Delta_{M}}} \notin \partial^{+} \Delta_{M}\right]<0 .
$$

Therefore, one can find a constant $\widetilde{c}$ so that for all large $M$ :

$$
P_{0}\left[X_{T_{\Delta_{M}}} \notin \partial^{+} \Delta_{M}\right]<e^{-\widetilde{c} M} .
$$

Furthermore, by taking the intersection of the set $\Delta_{M}$ with $\left\{x \in \mathbb{Z}^{d}: x \cdot l_{0}<\right.$ $M\}$, without loss of generality we can and do assume that $\Delta_{M} \subset\left\{x \in \mathbb{Z}^{d}\right.$ : $\left.x \cdot l_{0}<M\right\}$. Consider the box $\widetilde{B}_{M, \hat{r}, b, l_{0}}(0)$ defined by

$$
\widetilde{B}_{M, r, b, l_{0}}(0)=\widetilde{R}\left((-b M, M) \times(-\hat{r} M, \hat{r} M)^{d-1}\right),
$$

where $\widetilde{R}$ is a rotation on $\mathbb{R}^{d}$ with $\widetilde{R}\left(l_{0}\right)=e_{1}$. We have $\Delta_{M} \subset \widetilde{B}_{M, \hat{r}, b, l_{0}}(0)$, and consequently for large $M$,

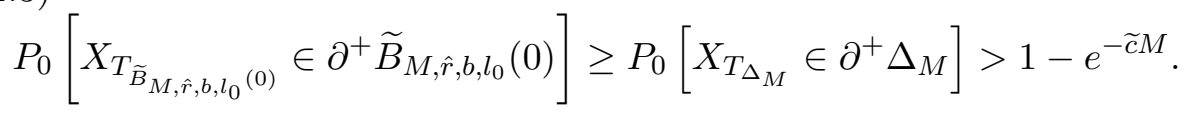

Notice that if $b \leq 1$, we choose $\mathfrak{r}$ in (iii) as $r$, and we finish the proof. Otherwise, we can proceed as follows: we take $N=b M$ and consider the box $B_{N, \hat{r}([b]+1) N, l_{0}}(0)$. We introduce for integer $i \in[1,[b]]$, a sequence $\left(T_{i}\right)_{1 \leq i \leq[b]}$ of $\left(\mathcal{F}_{n}\right)_{n \geq 0}$-stopping times via

$$
\begin{aligned}
& T_{1}=T_{\widetilde{B}_{M, \hat{r}, b, l_{0}}(0)}, \quad \text { and for } i>1 \\
& T_{i}=T_{\widetilde{B}_{M, \hat{r}, b, l_{0}}(0)} \circ \theta_{T_{i-1}}+T_{i-1} .
\end{aligned}
$$

As a result, we have

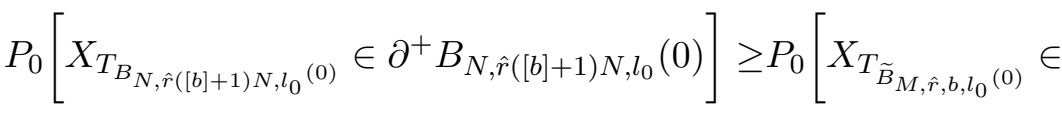

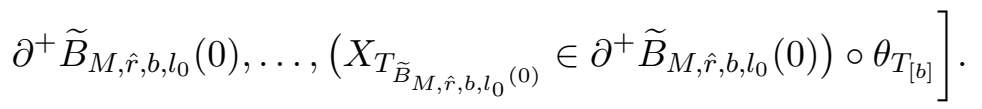

imsart-aop ver. 2014/10/16 file: trenormrev.tex date: November 19, 2018 
It is convenient at this point to introduce boundary sets $F_{i}, i \in[1,[b]]$ as follows:

$$
\begin{aligned}
F_{1} & =\partial^{+} B_{M, \hat{r}, b, l_{0}}(0) \text { and for } i>1 \\
F_{i} & =\bigcup_{y \in F_{i-1}} \partial^{+} B_{M, \hat{r}, b, l_{0}}(y)
\end{aligned}
$$

where $B_{M, \hat{r}, b, l_{0}}(y):=y+B_{M, \hat{r}, b, l_{0}}(0)$. We also introduce for $i \in[1,[b]]$, environment events $G_{i}$ via

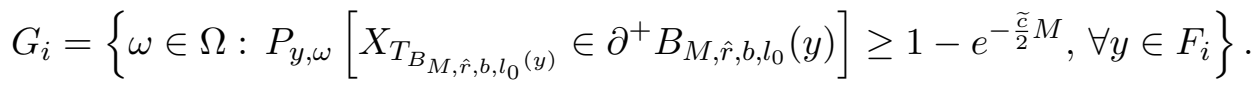

Observe that the right-hand side of inequality (2.5) is greater than

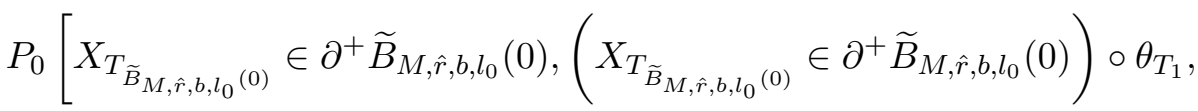

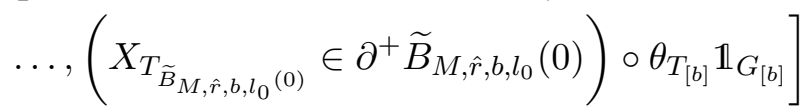

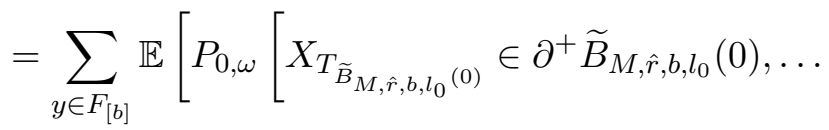

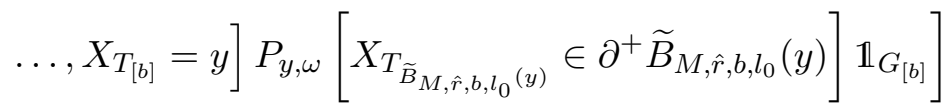

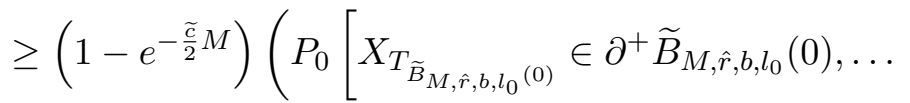

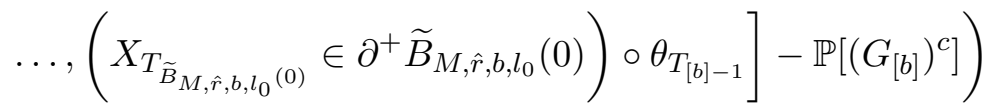

where we have made use of the Markov property. Iterate this argument recursively to obtain:

$$
\begin{aligned}
& P_{0}\left[X_{T_{B_{N, \hat{r}([b]+1) N, l_{0}}(0)}} \in \partial^{+} B_{N, \hat{r}([b]+1) N, l_{0}}(0)\right] \\
& \quad \geq\left(1-e^{-\frac{\tilde{c} M}{2}}\right)^{[b]+1}-\sum_{i=1}^{[b]}\left(1-e^{-\frac{\tilde{c} M}{2}}\right)^{[b]-i} \mathbb{P}\left[\left(G_{i}\right)^{c}\right] .
\end{aligned}
$$

Notice that using (2.3) along with Chebysev's inequality, we have for $i \in$ $[1,[b]]$ and large $M$,

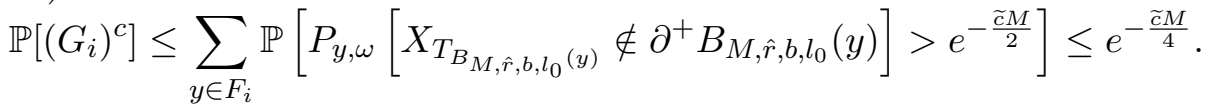

$$
\begin{aligned}
& \text { imsart-aop ver. 2014/10/16 file: trenormrev.tex date: November 19, } 2018
\end{aligned}
$$


From (2.6), the fact that $b$ is finite and independent of $M$ and the estimate (2.7); there exists a constant $w>0$, so that for large $N$

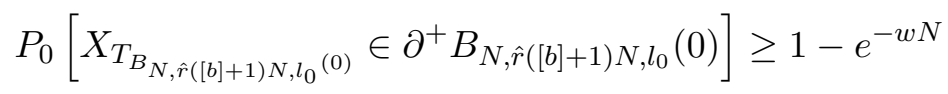

and this ends the proof of the implication (ii) implies (iii) by taking $\mathfrak{r}=$ $\hat{r}([b]+1)$.

To prove the implication (iii) $\Rightarrow$ (i), we fix a rotation $R$ on $\mathbb{R}^{d}$, with $R\left(e_{1}\right)=l_{0}$ and such that $R$ is the underlying rotation of hypothesis in (iii). For small $\alpha$ we define $2(d-1)$-directions $l_{+i}$ and $l_{-i}, i \in[2, d]$

$$
l_{+i}=\frac{l_{0}+\alpha R\left(e_{i}\right)}{\left|l_{0}+\alpha R\left(e_{i}\right)\right|_{2}} \text { and } l_{-i}=\frac{l_{0}-\alpha R\left(e_{i}\right)}{\left|l_{0}-\alpha R\left(e_{i}\right)\right|_{2}} .
$$

Following the same type of argument as in [GR17], Proposition 4.2, pp 1315 ; but using exponential decay instead of polynomial one; we conclude that there exists a small and positive $\alpha$, so that for each $i \in[2, d]$ there are some $r_{i}>0$, with

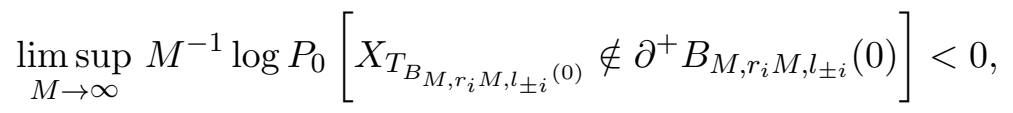

Thus, (2.8) finishes the proof by taking

$$
\begin{aligned}
& a_{0}=1, a_{1}=a_{2}=\ldots=a_{2(d-1)}=\frac{1}{2}, \\
& b_{0}=b_{1}=\ldots=b_{2(d-1)}=1, \\
& l_{0}, l_{1}=l_{+1}, l_{2}=l_{-1}, \ldots, l_{2(d-1)-1}=l_{+(d-1)}, l_{2(d-1)}=l_{-(d-1)},
\end{aligned}
$$

and then observing that for integer $i \in[0,2(d-1)]$

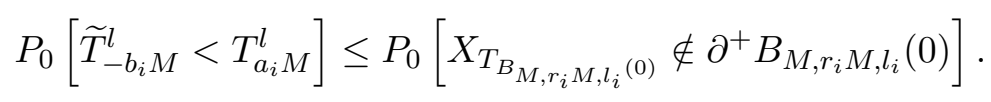

2.2. Approximate Renewal Structure. Throughout this section we assume that condition $(\mathbf{T})_{\ell}$ holds, where $\ell \in \mathbb{S}^{d-1}$. We observe that one can and does assume $\ell$ so that there exists $h \in(0, \infty)$ with $h \ell=: l \in \mathbb{Z}^{d}$. This is not a further restriction since by item $i$ ) of Lemma 2.2 , the set $B \subset \mathbb{S}^{d-1}$ of directions $\ell \in B$ such that $(\mathbf{T})_{\ell}$ holds contains an open set, thus writing

$$
A=\left\{u \in \mathbb{S}^{d-1}: \exists t \in(0, \infty) \text { with } t u \in \mathbb{Z}^{d}\right\} .
$$

imsart-aop ver. 2014/10/16 file: trenormrev.tex date: November 19, 2018 
one has that $A$ is dense in $\mathbb{S}^{d-1}$. Therefore we assume condition $(\mathbf{T})_{\ell}$, where $\ell$ is as above and choose a fixed $h>0$ with

$$
l:=h \ell \in \mathbb{Z}^{d} .
$$

We will denote the canonical orthonormal basis by $e_{i}, i \in[1, d]$ and consider the probability measure $\bar{P}_{0}$ given by

$$
\bar{P}_{0}:=\mathbb{P} \otimes Q \otimes P_{\omega, \varepsilon}^{0} \text { on } \Omega \times(\mathcal{W})^{\mathbb{N}} \times\left(\mathbb{Z}^{d}\right)^{\mathbb{N}},
$$

where $\mathcal{W}=\left\{z: z= \pm e_{i}\right.$, for some $\left.i \in[1, d]\right\} \cup\{0\}$, which is defined as follows: $Q$ is a product probability measure such that with each sequence $\varepsilon=\left(\varepsilon_{1}, \varepsilon_{2}, \ldots\right) \in(\mathcal{W})^{\mathbb{N}}$, for $i \in[1, d]$ we have $Q\left[\varepsilon_{1}= \pm e_{i}\right]=\kappa$ and $Q\left[\varepsilon_{1}=\right.$ $0]=1-2 d \kappa$. Then for fixed random elements $\varepsilon \in(\mathcal{W})^{\mathbb{N}}$ and $\omega \in \Omega$, we define $P_{\omega, \varepsilon}^{0}$ as the law of the Markov chain $\left(X_{n}\right)_{n \geq 0}$ with state space in $\mathbb{Z}^{d}$, starting from $0 \in \mathbb{R}^{d}$ and transition probabilities

$$
P_{\omega, \varepsilon}^{0}\left[X_{n+1}=X_{n}+e \mid X_{n}\right]=\mathbb{1}_{\left\{\varepsilon_{n+1}=e\right\}}+\frac{\mathbb{1}_{\left\{\varepsilon_{n+1}=0\right\}}}{1-2 d \kappa}\left(\omega\left(X_{n}, e\right)-\kappa\right),
$$

where $e$ is an element of the set $\left\{y \in \mathbb{Z}^{d}:|y|_{2}=1\right\}$. The importance of this auxiliary probability space stems from the easy to verify fact that the law of $\left(X_{n}\right)_{n \geq 0}$ under $Q \otimes P_{\omega, \varepsilon}^{0}$ coincides with the law under $P_{0, \omega}$, while the law under $\mathbb{P} \otimes P_{\omega, \varepsilon}^{0}$ coincides with $P_{0}$.

Define now the sequence $\bar{\varepsilon}$ of length $|l|_{1}$ in the following way: $\bar{\varepsilon}_{1}=\bar{\varepsilon}_{2}=$ $\ldots=\bar{\varepsilon}_{\left|l_{1}\right|}=\operatorname{sign}\left(l_{1}\right) e_{1}, \bar{\varepsilon}_{\left|l_{1}\right|+1}=\bar{\varepsilon}_{\left|l_{1}\right|+2}=\ldots=\bar{\varepsilon}_{\left|l_{1}\right|+\left|l_{2}\right|}=\operatorname{sign}\left(l_{2}\right) e_{2}$, $\ldots, \bar{\varepsilon}_{|l|_{1}-\left|l_{d}\right|+1}=\ldots=\bar{\varepsilon}_{|l|_{1}}=\operatorname{sign}\left(l_{d}\right) e_{d}$. Define for $\zeta>0$ small, $x \in \mathbb{Z}^{d}$, the cone $C(x, l, \zeta)$ by

$$
C(x, l, \zeta):=\left\{y \in \mathbb{Z}^{d}:(y-x) \cdot l \geq \zeta|l|_{2}|y-x|_{2}\right\} .
$$

We will assume that $\zeta$ is small enough in order to satisfy the following requirement:

$$
\bar{\varepsilon}_{1}, \bar{\varepsilon}_{1}+\bar{\varepsilon}_{2}, \ldots, \bar{\varepsilon}_{1}+\bar{\varepsilon}_{2}+\ldots+\bar{\varepsilon}_{|l|_{1}} \in C(0, l, \zeta) .
$$

For $L \in|l|_{1} \mathbb{N}$ we will denote by $\bar{\varepsilon}^{(L)}$ the vector

$$
\bar{\varepsilon}^{(L)}=\overbrace{(\bar{\varepsilon}, \bar{\varepsilon}, \ldots, \bar{\varepsilon}, \bar{\varepsilon})}^{L /|l|_{1}-\text { times }}
$$

of length equal to $L$. Setting

$$
D^{\prime}:=\inf \left\{n \geq 0: X_{n} \notin C\left(X_{0}, l, \zeta\right)\right\},
$$

we have: 
Lemma 2.3. Assume condition $(\boldsymbol{T})_{\ell}$, and fix $\mathfrak{r}$ and a rotation $R$ as in item iii) of Lemma 2.2. Then there exists $c_{1}>0$ such that if $\zeta<$ $\min \left\{\frac{1}{9 d}, \frac{1}{3 d \mathfrak{v}}\right\}$, then

$$
P_{0}\left[D^{\prime}=\infty\right] \geq c_{1}
$$

Proof. For $x \in \mathbb{Z}^{d}$ and $\alpha>0$, we define the flat cone $\mathcal{C}(x, \alpha, \ell)$ by

$$
\begin{aligned}
\mathcal{C}(x, \alpha, \ell)= & \left\{y \in \mathbb{Z}^{d}:(y-x) \cdot \frac{\ell+\alpha R\left(e_{i}\right)}{\left|\ell+\alpha R\left(e_{i}\right)\right|_{2}} \geq 0,\right. \\
& \left.(y-x) \cdot \frac{\ell-\alpha R\left(e_{i}\right)}{\left|\ell-\alpha R\left(e_{i}\right)\right|_{2}} \geq 0, \forall i \in[2, d]\right\} .
\end{aligned}
$$

It is clear when $y \in \mathcal{C}(x, \alpha, \ell)$, using the fact that for $i \in[2, d],\left|\ell \pm \alpha R\left(e_{i}\right)\right|_{2}>$ 0 (since $R\left(e_{1}\right)=\ell$ ), if $\alpha<1$ one has for all $i \in[2, d]$ :

$$
\begin{aligned}
& (y-x) \cdot l \geq \alpha\left|(y-x) \cdot R\left(e_{i}\right)\right| \\
& (y-x) \cdot l \geq \frac{\alpha}{d} \sum_{i=1}^{d}\left|(y-x) \cdot R\left(e_{i}\right)\right| \geq \frac{\alpha}{d}|y-x|_{2} .
\end{aligned}
$$

As a result $\mathcal{C}(x, \alpha, \ell) \subset C\left(x, \ell, \frac{\alpha}{d}\right)=C\left(x, l, \frac{\alpha}{d}\right)$. On the other hand, the polynomial condition ( $W P$ ) of [GR17] page 11, is obviously implied by $i i i)$ of Lemma 2.2. We finish the proof by applying Proposition 5.1 of [GR17].

We choose $\zeta>0$ satisfying the hypotheses of Lemma 2.3. For each $L \in$ $|l|_{1} \mathbb{N}$, we define $S_{0}=0$, and denoting by $\theta$ the canonical time shift, we set

$$
\begin{aligned}
& S_{1}=\inf \left\{n \geq L: X_{n-L} \cdot l>\max _{0 \leq j<n-L}\left\{X_{j} \cdot l\right\},\left(\varepsilon_{n-L}, \ldots, \varepsilon_{n-1}\right)=\bar{\varepsilon}^{(L)}\right\}, \\
& R_{1}=D^{\prime} \circ \theta_{S_{1}}+S_{1},
\end{aligned}
$$

and for $n>1$

$$
\begin{aligned}
& S_{n} \\
& \quad=\inf \left\{n>R_{n-1}: X_{n-L} \cdot l>\max _{0 \leq j<n-L}\left\{X_{j} \cdot l\right\},\left(\varepsilon_{n-L}, \ldots, \varepsilon_{n-1}\right)=\bar{\varepsilon}^{(L)}\right\}, \\
& R_{n}=D^{\prime} \circ \theta_{S_{n}}+S_{n},
\end{aligned}
$$

where we define $S_{n}=\infty$ or $R_{n}=\infty$ whenever the respective previous random variable is $\infty$. For given $L$ as above, these random variables are stopping times for the canonical underlying filtration of the pair $\left(X_{n}, \varepsilon_{n}\right)_{n \geq 0}$. Notice also that the chain of inequalities

$$
S_{0}=0<S_{1} \leq R_{1} \leq \ldots \leq S_{n} \leq R_{n} \ldots \leq \infty
$$

imsart-aop ver. 2014/10/16 file: trenormrev.tex date: November 19, 2018 
is satisfied, with strict inequality if the left member is finite. Indeed, we shall see in brief that under assumption $(\mathbf{T})_{\ell}$ all of them are strict inequalities. Setting

$$
K:=\inf \left\{n \geq 1: S_{n}<\infty, R_{n}=\infty\right\},
$$

one defines the first time of asymptotic regeneration $\tau_{1}:=S_{K} \leq \infty$ (we shall drop $L$ from the notation when there is not risk of confusion). A qualitative characterization of the time $\tau_{1}=n$ is as follows: the first time $n$ that the walk takes a strict record level in direction $l$ at time $n-L$, after which the walk is pushing through direction $l$ by unit steps on the lattice $\mathbb{Z}^{d}$ just owed to the action of $\bar{\varepsilon}^{(L)}$ sequence in the probability space $\left(Q,(\mathcal{W})^{\mathbb{N}}\right)$, independently on the environment, and finally for any future $j>n$ the walk remains forever inside the cone $C\left(X_{n}, l, \zeta\right)$.

The next lemma shows that the previous construction is significant and its proof can be derived from Lemma 2.3 in conjunction with the argument given in [Sz02], page 517 .

Lemma 2.4. Assume $(\mathbf{T})_{\ell}$. Then $P_{0}$-a.s. (see (2.9))

$$
\lim _{n \rightarrow \infty} X_{n} \cdot l=\infty
$$

and there exists a deterministic $L_{0}>0$, so that for each $L \geq L_{0}$, with $L \in|l|_{1} \mathbb{N}$, one has $\bar{P}_{0}$-a.s.

$$
\tau_{1}^{(L)}<\infty
$$

Choosing $L$ and $\zeta$ as prescribed by Lemmas 2.3-2.4, one has that $\bar{P}_{0}$-a.s. $\left\{R_{k}<\infty\right\}=\left\{S_{k+1}<\infty\right\}$ and $S_{1}<\infty$ by $(2.12)$.

Let us now define the iterates regeneration times of $\tau_{1}$ via:

$$
\tau_{n}=\tau_{1} \circ \theta_{\tau_{n-1}}+\tau_{n-1}
$$

for $n>1$. It is easy to verify that for any $k \in \mathbb{N}, \bar{P}_{0}-$ a.s. $\tau_{k}<\infty$.

The main technical objective of this article will be to obtain upper bounds for the $L$ dependent probabilities

$$
\bar{P}_{0}\left[\tau_{1}>u\right]
$$

where $u$ is large and independent on a fixed $L$. 
2.2.1. General Proof Strategy. From the fact that the proof of our main result Theorem 1.3 is a bit involved, we shall explain the general strategy to follow. Roughly speaking, we will try to recover all of the Sznitman's results of [Sz00] to bound the probability of the asymptotic regeneration time tails and then applying a version of the central limit theorem in [CZ02] we will obtain the proof. However, extending these results to the strong mixing case will prove to be technically more challenging.

3. On the Almost Renewal Structure for Random Walks in Strong Mixing Environments. Our mixing assumptions provide an approximate renewal structure when one considers the increments of the $\tau_{1}$ iterates. More precisely, we let $x \mathbb{Z}^{d}$ and $L \in|l|_{1} \mathbb{N}$ and define the $\sigma$-algebra:

$$
\mathcal{G}_{1}:=\sigma\left(\omega(y, \cdot): y \cdot l<X_{\tau_{1}} \cdot l-\frac{L|l|_{2}}{|l|_{1}},\left(\varepsilon_{i}\right)_{0 \leq i \leq \tau_{1}},\left(X_{i}\right)_{0 \leq i \leq \tau_{1}}\right),
$$

along with the random environment $\sigma$-algebra

$$
\mathfrak{F}_{x, L}:=\sigma\left(\omega(y, \cdot):(y-x) \cdot l \leq-\frac{L|l|_{2}}{|l|_{1}}\right) .
$$

An important technical fact comes in the next

Proposition 3.1 (Under either: $(\mathbf{S M})_{C, g}$ or $\left.(\mathbf{S M G})_{C, g}\right)$. For $L \in$ $|l|_{1} \mathbb{N}$ we let $\mu:=\mu(L)=\exp \left(e^{-g t L}\right)$. Then for each $t \in(0,1)$ there exists $L_{0}=L_{0}(C, g, \kappa, l, d, r) \in|l|_{1} \mathbb{N}$ such that $\bar{P}_{0}-$ a.s.

$$
\mu^{-1}(L) \bar{P}_{0}\left[\left(X_{n}-X_{0}\right)_{n \geq 0} \in \cdot \mid D^{\prime}=\infty\right] \leq \bar{P}_{0}\left[\left(X_{\tau_{1}+n}-X_{\tau_{1}}\right)_{n \geq 0} \in \cdot \mid \mathcal{G}_{1}\right]
$$

$$
\leq \mu(L) \bar{P}_{0}\left[\left(X_{n}-X_{0}\right)_{n \geq 0} \in \cdot \mid D^{\prime}=\infty\right]
$$

holds, for all $L \geq L_{0}, L \in|l|_{1} \mathbb{N}$.

Proof. We fix $t$ as in the statement of the proposition and consider non-negative bounded functions $f$ and $h$ which are $\sigma\left(\left(X_{n}\right)_{n \geq 0}\right)$ and $\mathcal{G}_{1}$ measurable, respectively. Denoting by $\vartheta$ and $\theta$ the space and time shifts, from the very definition of the renewal structure one has,

$$
\begin{aligned}
& \bar{E}_{0}\left[f\left(X_{\tau_{1}+\cdot}-X_{\tau_{1}}\right) h\right]=\sum_{k \geq 1} \bar{E}_{0}\left[f\left(X_{S_{k}+\cdot}-X_{S_{k}}\right) h, S_{k}<\infty, R_{k}=\infty\right] \\
= & \sum_{k \geq 1, j \geq 1, x \in \mathbb{Z}^{d}} \bar{E}_{0}\left[f\left(X_{S_{k}+\cdot}-x\right) h, X_{S_{k}}=x, S_{k}=j, D^{\prime} \circ \theta_{n}=\infty\right] .
\end{aligned}
$$


Observe that over the event $\left\{X_{S_{k}}=x, S_{k}=j, D^{\prime} \circ \theta_{j}=\infty\right\}$ one can find a bounded function $h_{x, k, j}$, which is $\sigma\left(\left(\omega(y, \cdot), y \cdot l<x \cdot l-\left(L|l|_{2}\right) /\left(|l|_{1}\right)\right) \otimes\right.$ $\left.\left(X_{n}\right)_{0 \leq n \leq j}\right)$-measurable and equal to $h$. As a result, the rightmost term in the previous display equals

$$
\sum_{k, j \geq 1, x \in \mathbb{Z}^{d}} \mathbb{E}\left[E_{Q \otimes P_{\varepsilon, \omega}^{0}}\left[f\left(X_{S_{k}+\cdot}-x\right) h_{x, k, j}, X_{S_{k}}=x, S_{k}=j, D^{\prime} \circ \theta_{n}=\infty\right]\right]
$$

Applying now the strong Markov property at time $S_{k}$ and using the product structure of $Q$ one sees in turn that equals

$$
\begin{array}{rl}
\sum_{k, j \geq 1, x \in \mathbb{Z}^{d}} & \mathbb{E}\left[E_{Q \otimes P_{\varepsilon, \omega}^{0}}\left[h_{x, k, j}, X_{S_{k}}=x, S_{k}=j\right]\right. \\
\times & \left.E_{Q \otimes P_{\vartheta_{n}, \theta_{x} \omega}^{0}}\left[f(X .-x), D^{\prime}=\infty\right]\right] .
\end{array}
$$

Use notation (3.1) to obtain that (3.3) equals

$$
\begin{array}{rl}
\sum_{k, j \geq 1, x \in \mathbb{Z}^{d}} & \mathbb{E}\left[E_{Q \otimes P_{\varepsilon, \omega}^{0}}\left[h_{x, k, j}, X_{S_{k}}=x, S_{k}=j\right]\right. \\
\times & \left.\mathbb{E}\left[E_{Q \otimes P_{\vartheta_{n \varepsilon}, \theta_{x} \omega}^{0}}\left[f\left(X .-X_{0}\right), D^{\prime}=\infty\right] \mid \mathfrak{F}_{x, L}\right]\right] .
\end{array}
$$

Fix $x \in \mathbb{Z}^{d}, n \in \mathbb{N}$ and consider the conditional probability distribution

$$
\hat{\mathbb{P}}\left[\cdot \mid \mathfrak{F}_{x, L}\right]:=\frac{\mathbb{E}\left[P_{Q \otimes P_{\vartheta_{n \varepsilon}, \theta_{x} \omega}^{0}}\left[\left(X_{i}-X_{0}\right)_{i \geq 0} \in \cdot D^{\prime}=\infty\right] \mid \mathfrak{F}_{x, L}\right]}{\mathbb{E}\left[P_{Q \otimes P_{\vartheta_{n \varepsilon}, \theta_{x} \omega}^{0}}\left[D^{\prime}=\infty\right] \mid \mathfrak{F}_{x, L}\right]} .
$$

It will be proven below that there exists a positive constant $L_{0}>0$, so that for each $L \in|l|_{1} \mathbb{N}, L \geq L_{0}$ we have $\bar{P}_{0}$-a.s.

$$
\begin{aligned}
& \exp \left(-e^{-g t L}\right) \bar{P}_{0}\left[\left(X_{i}-X_{0}\right)_{i \geq 0} \in \cdot \mid D^{\prime}=\infty\right] \leq \hat{\mathbb{P}}\left[\cdot \mid \mathfrak{F}_{x, L}\right] \\
& \leq \exp \left(e^{-g t L}\right) \bar{P}_{0}\left[\left(X_{i}-X_{0}\right)_{i \geq 0} \in \cdot \mid D^{\prime}=\infty\right] .
\end{aligned}
$$

Thus using (3.5) and (2.13), writing (3.4) as

$$
\begin{gathered}
A=\sum_{k, j \geq 1, x \in \mathbb{Z}^{d}} \mathbb{E}\left[E_{Q \otimes P_{\varepsilon, \omega}^{0}}\left[h_{x, k, j}, X_{S_{k}}=x, S_{k}=j\right]\right. \\
\left.\times \mathbb{E}\left[P_{Q \otimes P_{\vartheta_{n} \varepsilon, \theta_{x} \omega}^{0}}\left[D^{\prime}=\infty\right] \mid \mathfrak{F}_{x, L}\right] \frac{\mathbb{E}\left[E_{Q \otimes P_{\vartheta_{n \varepsilon}, \theta_{x} \omega}^{0}}\left[f\left(X .-X_{0}\right), D^{\prime}=\infty\right] \mid \mathfrak{F}_{x, L}\right]}{\mathbb{E}\left[P_{Q \otimes P_{\vartheta_{n} \varepsilon, \theta_{x} \omega}^{0}}\left[D^{\prime}=\infty\right] \mid \mathfrak{F}_{x, L}\right]}\right] \\
\text { imsart-aop ver. 2014/10/16 file: trenormrev.tex date: November 19, } 2018
\end{gathered}
$$


one has

$$
\exp \left(-e^{-g t L}\right) \bar{E}_{0}[h] \bar{E}_{0}\left[f \mid D^{\prime}=\infty\right] \leq A \leq \exp \left(e^{-g t L}\right) \bar{E}_{0}[h] \bar{E}_{0}\left[f \mid D^{\prime}=\infty\right]
$$

which finishes the proof.

Let us now prove the claim (3.5). Our proof shares some similarities with the proofs of X. Guo in Lemma 5 and Proposition 7 of [Gu14].

Lemma 3.2. Under the assumptions and notation of Proposition 3.1. Let $x_{0} \in \mathbb{Z}^{d}$ and $n \in \mathbb{N}$, then there exists $L_{0}=L_{0}(C, g, \kappa, l, d, r) \in|l|_{1} \mathbb{N}$ such that

$$
\begin{aligned}
& \exp \left(-e^{-g t L}\right) \bar{P}_{0}\left[\left(X_{i}-X_{0}\right)_{i \geq 0} \in \cdot \mid D^{\prime}=\infty\right] \leq \hat{\mathbb{P}}\left[\cdot \mid \mathfrak{F}_{x_{0}, L}\right] \\
& \leq \exp \left(e^{-g t L}\right) \bar{P}_{0}\left[\left(X_{i}-X_{0}\right)_{i \geq 0} \in \cdot \mid D^{\prime}=\infty\right],
\end{aligned}
$$

for all $L \geq L_{0}$, with $L \in|l|_{1} \mathbb{N}$.

Proof. We split the proof into three steps.

Step 1. The first step is the following claim:

Let $A \subset \Lambda \subset \mathbb{Z}^{d}$. Suppose $S \neq \varnothing$ is a countable set of finite paths $x .=\left(x_{i}\right)_{i=0}^{N}, N<\infty$ starting at $x_{0}$ that satisfy $d_{1}(x ., \Lambda) \geq r$ and

$$
\sum_{y \in A, 0 \leq i \leq N} e^{-g\left|y-x_{i}\right|_{1}} \leq a
$$

uniformly on $N$. Then $\mathbb{P}$-a.s. (cf. [Gu14], page 381 for a proof)

$$
\begin{aligned}
& \exp (-C a) \\
& \leq \frac{\mathbb{E}\left[E_{Q}\left[P_{\omega \circ \theta_{x_{0}}, \varepsilon \circ \vartheta_{n}}\left[\bigcup_{N \geq 0}\left\{\left(X_{i}-X_{0}\right)_{0 \leq i \leq N} \in S\right\}\right]\right] \mid \omega_{y}, y \in \Lambda\right]}{\mathbb{E}\left[E_{Q}\left[P_{\omega \circ \theta_{x_{0}}, \varepsilon \circ \vartheta_{n}}\left[\bigcup_{N \geq 0}\left\{\left(X_{i}-X_{0}\right)_{0 \leq i \leq N} \in S\right\}\right]\right] \mid \omega_{y}, y \in \Lambda \backslash A\right]} \\
& \leq \exp (C a) .
\end{aligned}
$$

Step 2. Consider the hyperplane $H_{L, l}$ defined by

$$
H_{L, l}:=\left\{z \in \mathbb{Z}^{d}: z \cdot l \leq-\left(L|l|_{2}\right) /|l|_{1}\right\} .
$$

In this step, we will first estimate the series

$$
\begin{aligned}
& \sum_{\substack{y \in \partial^{r} H_{L, l}, z \in \partial^{r} C(0, l, \zeta)}} \exp \left(-g|y-z|_{1}\right) \quad \text { and } \\
& \sum_{\substack{y \in H_{L, l} \\
z \in C(0, l, \zeta)}} \exp \left(-g|y-z|_{1}\right)
\end{aligned}
$$


in terms of $g$, for some large but fixed $L$. Notice that for given $L>0$, both series in (3.7) converge because $\zeta>0$, as follows from the next argument. Choose $\hat{t} \in(t, 1)$ and consider the first series in (3.7). We take $L$ large enough so that $L>(1-\hat{t})^{-1} 2 r$ (thus $L-2 r>\hat{t} L$ ) and applying condition $(\mathbf{S M})_{C, g}$.

$$
\sum_{n \geq 0} \sum_{(y, z) \in \mathcal{H C}_{L, n, y, z}} e^{-g|y-z|_{1}},
$$

where we have written

$$
\begin{aligned}
& \mathcal{H C}_{L, n, y, z}:=\left\{(y, z): y \in \partial^{r} H_{L, l}, z \in \partial^{r} C(0, l, \zeta),\right. \\
& \left.\hat{t} L+n \leq|y-z|_{1} \leq \hat{t} L+(n+1)\right\}
\end{aligned}
$$

Above was used the fact that the minimal $|\cdot|{ }_{1}$-distance between any two points $y \in \partial^{r} H_{L, l}, z \in \partial^{r} C(0, l, \zeta)$ is at least $L-2 r$.

Therefore we obtain the following upper bound for series (3.7):

$$
\sum_{n \geq 0}\left|H_{L, n, y, z}\right| e^{-g(\hat{t} L+n)}
$$

On the other hand, the estimate

$$
\left|H_{L, n, y, z}\right| \leq \tilde{c}(d) r^{2}(n+1)^{2(d-1)}
$$

holds, for a suitable $\tilde{c}>0$ depending on $d$ and $\zeta$. Notice also that

$$
\sum_{n \geq 0}(n+1)^{2(d-1)} e^{-g n}
$$

converges, thus combining both last estimates we conclude: there exists $C_{1}=C_{1}(C, d, g, r, \zeta, l)>0$ scuh that if $L \geq C_{1}$ one can bound from above series (3.7) by

$$
\exp (-g \widetilde{t} L)
$$

where $\tilde{t} \in(t, \hat{t})$.

Performing the same type of argument, one sees that from the fact that the inner angle of the cone is positive there exists $C_{2}>0$, so that:

$$
\sum_{y \in H_{L, l}, z \in C(0, l, \zeta)} \exp \left(-g|y-z|_{1}\right) \leq \exp (-g t L)
$$

holds, for all $L \in \mathbb{N}|l|_{1}, L \geq L_{0}$, provided that $L_{0} \geq C_{2}$.

imsart-aop ver. 2014/10/16 file: trenormrev.tex date: November 19, 2018 
Consequently, for a given finite path starting from $x_{0}$ of the form

$$
x .=\left(X_{i}\right)_{i=0}^{N}, N<\infty, x . \subset C\left(x_{0}, l, \zeta\right)
$$

one has that uniformly on $N$, there exists a positive constant $C_{3}$ such that if $L \geq C_{3}$

$$
\sum_{y \in \partial^{r} H_{L, l, x_{0}}, z \in \partial^{r} G_{x}} \exp \left(-g\left|y-\left(z-x_{0}\right)\right|_{1}\right) \leq e^{-g \widetilde{t} L},
$$

provided that we define

$$
H_{L, l, x_{0}}:=\left\{z \in \mathbb{Z}^{d}:\left(z-x_{0}\right) \cdot l \leq-\left(L|l|_{2} /\left(|l|_{1}\right)\right)\right\}
$$

and

$$
G_{x}:=\left\{y \in \mathbb{Z}^{d}: y=X_{i}, \text { for some } i \in[0, N]\right\} .
$$

Likewise using the second estimate in (3.9), we obtain a suitable constant $C_{4}$ such that $L_{0} \geq C_{4}$ implies that

$$
\sum_{y \in H_{L, l, x_{0}}, 0 \leq i \leq N} \exp \left(-g\left|y-\left(X_{i}-x_{0}\right)\right|_{1}\right) \leq e^{-g \widetilde{t} L}
$$

holds, for $L \geq L_{0}$, uniformly on $N \in \mathbb{N}$, where the notation is as above. We then consider, instead of a fixed path $x$, a countable collection $S$ of finite paths starting from a common point $x_{0} \in \mathbb{Z}^{d}$ with all of them contained in a cone $C\left(x_{0}, l, \zeta\right)$. Therefore, choosing $\widehat{t} \in(t, \widetilde{t})$ we find that there exists $C_{5}$ so that whenever $L \geq C_{5}$, Step 1 gives

$$
\begin{aligned}
& \exp \left(-e^{-g \widehat{t} L}\right) \\
& \leq \frac{\mathbb{E}\left[E_{Q}\left[P_{\omega \circ \theta_{x_{0}}, \varepsilon \circ \vartheta_{n}}\left[\bigcup_{N \geq 0}\left\{\left(X_{i}-X_{0}\right)_{0 \leq i \leq N} \in S\right\}\right]\right] \mid \omega_{y}, y \in \Lambda\right]}{\mathbb{E}\left[E_{Q}\left[P_{\omega \circ \theta_{x_{0}}, \varepsilon \circ \vartheta_{n}}\left[\bigcup_{N \geq 0}\left\{\left(X_{i}-X_{0}\right)_{0 \leq i \leq N} \in S\right\}\right]\right] \mid \omega_{y}, y \in \Lambda \backslash A\right]} \\
& \leq \exp \left(e^{-g \widehat{t} L}\right),
\end{aligned}
$$

where $\Lambda=H_{L, l, x_{0}}$, and $A$ is an arbitrary subset of $\Lambda$.

Step 3. We prove here the assertion of the lemma. For $j \in \mathbb{N}$, we set $S_{0, j}$ the set of paths of length $j-1$ starting from 0 . Then by definition one has

$$
\begin{aligned}
& \left\{\left(X_{i}-X_{0}\right)_{i \geq 0} \in \cdot, D^{\prime}=\infty\right\} \\
& =\bigcap_{n \geq 0} \bigcup_{N \geq 0} \bigcup_{j=0}^{N}\left\{\left(X_{i}-X_{0}\right)_{i=0}^{j} \in S_{0, j}, D^{\prime}>n\right\} .
\end{aligned}
$$

imsart-aop ver. 2014/10/16 file: trenormrev.tex date: November 19, 2018 
For any $n \in \mathbb{N}$, an application of Step 1 and Step 2 lead us to

$$
\begin{aligned}
& \exp \left(-e^{-g \widehat{t} L}\right) \\
& \leq \frac{\mathbb{E}\left[E_{Q}\left[P_{\omega \circ \theta_{x_{0}}, \varepsilon \circ \vartheta_{n}}\left[\left(X_{i}-X_{0}\right)_{i \geq 0} \in \cdot, D^{\prime}>n\right]\right] \mid \omega_{y}, y \in \Lambda\right]}{\mathbb{E}\left[E_{Q}\left[P_{\omega \circ \theta_{x_{0}}, \varepsilon \circ \vartheta_{n}}\left[\left(X_{i}-X_{0}\right)_{i \geq 0} \in \cdot D^{\prime}>n\right]\right] \mid \omega_{y}, y \in \Lambda \backslash A\right]} \\
& \leq \exp \left(e^{-g \widehat{t} L}\right),
\end{aligned}
$$

where $\Lambda$ and $A$ are as in Step $\mathcal{Z}$ (recall that $A$ is an arbitrary subset of $\Lambda$ ). Letting $n \rightarrow \infty$ and then using the result for $A=\Lambda$, one gets

$$
\begin{aligned}
& \exp \left(-e^{-g \widehat{t} L}\right) \\
& \leq \frac{\mathbb{E}\left[E_{Q}\left[P_{\omega \circ \theta_{x_{0}}, \varepsilon \circ \vartheta_{n}}\left[\left(X_{i}-X_{0}\right)_{i \geq 0} \in \cdot, D^{\prime}=\infty\right]\right] \mid \omega_{y}, y \in \Lambda\right]}{P_{0}\left[\left(X_{i}-X_{0}\right)_{i \geq 0} \in \cdot, D^{\prime}=\infty\right]} \\
& \leq \exp \left(e^{-g \widehat{t} L}\right),
\end{aligned}
$$

and

$$
\begin{aligned}
& \exp \left(-e^{-g \widehat{t} L}\right) \\
& \leq \frac{\mathbb{E}\left[E_{Q}\left[P_{\omega \circ \theta_{x_{0}}, \varepsilon \circ \vartheta_{n}}\left[D^{\prime}=\infty\right]\right] \mid \omega_{y}, y \in \Lambda\right]}{P_{0}\left[D^{\prime}=\infty\right]} \leq \exp \left(e^{-g \widehat{t} L}\right) .
\end{aligned}
$$

By choosing $L_{0}$ large enough such that for $L \geq L_{0}$

$$
2 e^{-g \widehat{t} L} \leq e^{-g t L},
$$

we finish the proof.

We close this section with a straightforward consequence of the previous proposition which will be stated in the next corollary, for reference purposes. As a natural extension to $\mathcal{G}_{1}$, we define the sigma-algebra $\mathcal{G}_{i}$, where $i \in \mathbb{N}$, by

$$
\mathcal{G}_{i}=\sigma\left(\omega(y, \cdot): y \cdot l<X_{\tau_{i}} \cdot l-\left(L|l|_{2}\right) /\left(|l|_{1}\right),\left(\varepsilon_{i}\right)_{0 \leq j \leq \tau_{i}},\left(X_{j}\right)_{0 \leq j \leq \tau_{i}}\right) .
$$

Let $\mu$ be as in the statement of Proposition 3.1, then an induction argument makes us conclude: 
Corollary 3.3. Assume either: $(\boldsymbol{S M})_{C, g}$ or $(\boldsymbol{S} \boldsymbol{M G})_{C, g}$ and let $j \in$ $\mathbb{N}, t \in(0,1)$. Then there exists $L_{0}=L_{0}(C, g, \kappa, l, d, r) \in|l|_{1} \mathbb{N}$ such that $\mathbb{P}$-a.s.

$$
\begin{aligned}
& \mu^{-1}(L) \bar{P}_{0}\left[\left(X_{n}-X_{0}\right)_{n \geq 0} \in \cdot \mid D^{\prime}=\infty\right] \leq \bar{P}_{0}\left[\left(X_{\tau_{j}+n}-X_{\tau_{j}}\right)_{n \geq 0} \in \cdot \mid \mathcal{G}_{j}\right] \\
& \leq \mu(L) \bar{P}_{0}\left[\left(X_{n}-X_{0}\right)_{n \geq 0} \in \cdot \mid D^{\prime}=\infty\right]
\end{aligned}
$$

holds, for all $L \geq L_{0}$ with $L \in|l|_{1} \mathbb{N}$.

4. Preliminary Estimates: The Regeneration Position has some Exponential Moments. It is the purpose of this section to prove that the random variable $X_{\tau_{1}} \cdot l$ has some finite exponential moments under condition $(\mathbf{T})_{\ell}($ recall $(2.9))$. We will derive after that proof two further consequences. On the one hand it will be showed the finiteness of some exponential moments for the random variable $\sup _{0 \leq n \leq \tau_{1}}\left|X_{n}\right|_{2}$; and on the other hand, an upper bound of stretched exponential-type for the probability of large orthogonal oscillations along the approximate asymptotic direction of the walk. Throughout the rest of the paper we assume condition $(\mathbf{T})_{\ell}$, and we pick $h \in(0, \infty)$ so that $(2.9)$ is satisfied. Then we choose a constant $\mathfrak{r}>0$ as in the item iii) of Lemma 2.2 and the cone angle $\zeta$ will be any fixed positive number satisfying the following requirement

$$
\zeta<\min \left\{\frac{1}{9 d}, \frac{1}{3 d \mathfrak{r}}, \cos \left(\frac{\pi}{2}-\arctan (3 \mathfrak{r})\right)\right\} .
$$

Proposition 4.1. Assume that $(\boldsymbol{T})_{\ell}$ and either $(\boldsymbol{S M})_{C, g}$ or $(\boldsymbol{S M G})_{C, g}$ hold. Then there exist positive constants $c_{2}, c_{3}$ and $L_{0}$, such that for all $L \geq L_{0}$, with $L \in|l|_{1} \mathbb{N}$ :

$$
\bar{E}_{0}\left[\exp \left(c_{2} \kappa^{L} X_{\tau_{1}} \cdot l\right)\right]<c_{3}
$$

holds.

Proof. By virtue of the renewal structure definitions, for $c>0$ and $L \in|l|_{1} \mathbb{N}$, one has that:

$$
\begin{gathered}
\bar{E}_{0}\left[\exp \left(c \kappa^{L} X_{\tau_{1}} \cdot l\right)\right] \\
=\sum_{k \geq 1} \bar{E}_{0}\left[\exp \left(c \kappa^{L} X_{S_{k}} \cdot l\right), S_{k}<\infty, D^{\prime} \circ \theta_{S_{k}}=\infty\right] \\
=\sum_{x \in \mathbb{Z}^{d}, n \in \mathbb{N}, k \in \mathbb{N}} \mathbb{E}\left[E_{Q \times P_{\varepsilon, \omega}^{0}}\left[e^{c \kappa^{L} x \cdot l}, X_{S_{k}}=x, S_{k}=n\right] P_{\theta_{n} \varepsilon, \theta_{x} \omega}^{0}\left[D^{\prime}=\infty\right]\right] . \\
\text { imsart-aop ver. 2014/10/16 file: trenormrev.tex date: November 19, } 2018
\end{gathered}
$$


Notice that for $k \geq 1$, the Markov property implies that

$$
\begin{aligned}
& \bar{E}_{0}\left[\exp \left(c \kappa^{L} X_{S_{k}} \cdot l\right), S_{k}<\infty, D^{\prime} \circ \theta_{S_{k}}=\infty\right] \\
& =\sum_{x \in \mathbb{Z}^{d}, n \in \mathbb{N}} \mathbb{E}\left[E_{Q}\left[E_{P_{\varepsilon, \omega}^{0}}\left[\exp \left(c \kappa^{L} x \cdot l\right), X_{S_{k}}=x, S_{k}=n\right] P_{\theta_{n} \varepsilon, \theta_{x} \omega}^{0}\left[D^{\prime}=\infty\right]\right]\right] .
\end{aligned}
$$

Observe now that the random variables

$$
E_{P_{\varepsilon, \omega}^{0}}\left[\exp \left(c \kappa^{L} x \cdot l\right), X_{S_{k}}=x, S_{k}=n\right]
$$

and $P_{\theta_{n}, \theta_{x} \omega}^{0}\left[D^{\prime}=\infty\right]$ are: $\sigma\left(\varepsilon_{i}, i<n\right) \otimes \sigma\left(\omega(y, \cdot),(y-x) \cdot l \leq L|l|_{2} /|l|_{1}\right)$ and $\sigma\left(\varepsilon_{i}, i \geq n\right) \otimes \sigma(\omega(y, \cdot), y \in C(x, l, \zeta))$ measurable, respectively.

Therefore for $x \in \mathbb{Z}^{d}$, using the previously introduced notation $\mathfrak{F}_{x, L}$ (cf. (3.1)), the mixing condition $(\mathbf{S M})_{C, g}$ and the construction of the probability measure $\bar{P}_{0}$ we find an $L_{0}>0$ such that for all $L \geq L_{0}$, with $L \in|l|_{1} \mathbb{N}$, the rightmost term of (4.3) is less than

$$
\begin{aligned}
\mathbb{E}[ & \left.\left.E_{Q \otimes P_{\theta_{n} \varepsilon, \theta_{x} \omega}^{0}}\left[\mathbb{1}_{D^{\prime}=\infty}\right] \mid \mathfrak{F}_{x, L}\right]\right] \leq \bar{E}_{0}\left[\exp \left(c \kappa^{L} X_{S_{k}} \cdot l\right), S_{k}<\infty\right] \\
& \times \exp \left(C \sum_{x \in \partial^{r}\left(H^{c}\right), y \in \partial^{r}\left(\Lambda^{c}\right)} e^{-g|x-y|_{2}}\right) P_{0}\left[D^{\prime}=\infty\right],
\end{aligned}
$$

where $H$ and $\Lambda$ denote the sets $\left\{z \in \mathbb{Z}^{d}: z \cdot l \leq-L|l|_{2} /|l|_{1}\right\}$ and $C(0, l, \zeta)$ respectively. Since $\zeta>0$, the proof of Proposition 3.1 provides the existence of a constant $\widehat{c}>0$ so that

$$
\exp \left(C \sum_{x \in \partial^{r}\left(H^{c}\right), y \in \partial^{r}\left(\Lambda^{c}\right)} e^{-g|x-y|_{2}}\right) \leq \exp \left(e^{-\widehat{c} L}\right),
$$

with a similar upper bound under $(\mathbf{S M G})_{C, g}$. Going back to (4.3), we have

$$
\begin{aligned}
& \bar{E}_{0}\left[\exp \left(c \kappa^{L} X_{S_{k}} \cdot l\right), S_{k}<\infty, D^{\prime} \circ \theta_{S_{k}}=\infty\right] \\
& \left.\leq 2 \bar{E}_{0}\left[\exp \left(c \kappa^{L} X_{S_{k}} \cdot l\right), S_{k}<\infty\right] P_{0}\left[D^{\prime}=\infty\right]\right] .
\end{aligned}
$$

We now proceed with the same type of argument of [GR17], Subsection 6.2; so as to obtain a recursion for $k \geq 0$ of the expression

$$
\bar{E}_{0}\left[\exp \left(c \kappa^{L} X_{S_{k+1}} \cdot l\right), S_{k+1}<\infty\right] .
$$

To this end, it will be convenient to introduce the random variable

$$
M_{k}:=\sup _{0 \leq n \leq R_{k}} X_{n} \cdot l
$$

imsart-aop ver. 2014/10/16 file: trenormrev.tex date: November 19, 2018 
for $k \geq 0$ (with the convention $M_{0}=0$ ). We also introduce the sets parametrized by $k, n \in \mathbb{N}$ :

$$
A_{n, k}=\left\{\varepsilon \in W^{\mathbb{N}}:\left(\varepsilon_{t_{k}^{(n)}}, \varepsilon_{t_{k}^{(n)}+1}, \ldots, \varepsilon_{t_{k}^{(n)}+L-1}\right)=\bar{\varepsilon}^{(L)}\right\}
$$

and:

$$
B_{n, k}=\left\{\varepsilon \in W^{\mathbb{N}}:\left(\varepsilon_{t_{k}^{(j)}}, \varepsilon_{t_{k}^{(j)}+1}, \ldots, \varepsilon_{t_{k}^{(j)}+L-1}\right) \neq \bar{\varepsilon}^{(L)} \forall j \in[0, n-1]\right\} .
$$

As was mentioned in [GR17], pp. 25-26; denoting by $\bar{T}_{a}^{l}$ where $a \in \mathbb{R}$ the first time that the walk goes on strictly over level $a$ in direction $l$, i.e.

$$
\bar{T}_{a}^{l}=\inf \left\{n \geq 0: X_{n} \cdot l>a\right\},
$$

and by $\left(t_{k}^{(n)}\right)_{n \geq 0}$ the time sequence of successive maxima in direction $l$, defined recursively via:

$$
t_{k}^{(0)}=\bar{T}_{M_{k}}^{l}, \quad \text { and for } n \geq 1: t_{k}^{(n)}=\bar{T}_{X_{t_{k}(n-1)}^{l} l}^{l}
$$

one has the inclusion:

$$
\left\{S_{k+1}<\infty\right\} \subseteq \bigcup_{n \geq 0}\left\{t_{k}^{(n)}<\infty, B_{n, k}, A_{n, k}\right\}
$$

Furthermore, $\bar{P}_{0}$-a.s. on the event $B_{n, k} \cap A_{n, k}$ the identity

$$
S_{k+1}=t_{k}^{(n)}+L
$$

holds. As a result, we have for $k \geq 0$ the inequality:

$$
\begin{aligned}
& \bar{E}_{0}\left[\exp \left(c \kappa^{L} X_{S_{k+1}} \cdot l\right), S_{k+1}<\infty\right] \\
& \leq \sum_{0 \leq n \leq L^{2}-1} \bar{E}_{0}\left[\exp \left(c \kappa^{L} X_{S_{k+1}} \cdot l\right), t_{k}^{n}<\infty, B_{n, k}, A_{n, k}\right] \\
& +\sum_{n \geq L^{2}} \bar{E}_{0}\left[\exp \left(c \kappa^{L} X_{S_{k+1}} \cdot l\right), t_{k}^{n}<\infty, B_{n, k}, A_{n, k}\right] \\
& \leq 2 \sum_{n \geq L^{2}} \bar{E}_{0}\left[\exp \left(c \kappa^{L} X_{S_{k+1}} \cdot l\right), t_{k}^{n}<\infty, B_{n, k}, A_{n, k}\right]
\end{aligned}
$$

where the last inequality in (4.6) can be verified by inspecting the orders of $L$ in both sums. Moreover, one can find a positive constant $\bar{c}$ such that $\bar{P}_{0}$-a.s. on the event $\left\{t_{k}^{(n)}<\infty, B_{n, k}, A_{n, k}\right\}$

$$
X_{S_{k+1}} \cdot l \leq M_{k}+n|l|_{\infty}+\bar{c} L
$$

imsart-aop ver. 2014/10/16 file: trenormrev.tex date: November 19, 2018 
holds. Using the product structure of the measure $Q$ and inequality (4.7), it follows that for $n \geq L^{2}$

$$
\begin{aligned}
& \bar{E}_{0}\left[\exp \left(c \kappa^{L} X_{S_{k+1}} \cdot l\right), t_{k}^{(n)}<\infty, B_{n, k}, A_{n, k}\right] \\
& \leq \kappa^{L} \bar{E}_{0}\left[\exp \left(c \kappa^{L}\left(M_{k}+n|l|_{\infty}+\bar{c} L\right)\right), t_{k}^{(n)}<\infty, B_{n, k}\right] .
\end{aligned}
$$

We now apply the Markov property at times $t_{k}^{(0)}$ and $t_{k}^{(n)}$ (recall that $n \geq$ $L^{2}$ ), together with Lemma 6.6 of [GR17] to see that for some positive constant $\widetilde{c}$, the inequality:

$$
\begin{aligned}
& \kappa^{L} \bar{E}_{0}\left[\exp \left(c \kappa^{L}\left(M_{k}+n|l|_{\infty}+\bar{c} L\right)\right), t_{k}^{n}<\infty, B_{n, k}\right] \\
& \leq 2 \kappa^{L}\left(\exp \left(c|l|_{\infty} \kappa^{L} L^{2}\right)\left(1-\widetilde{c} L^{2} \kappa^{L}\right)\right)^{\left[\frac{n}{L^{2}}\right]} \bar{E}_{0}\left[\exp \left(c \kappa^{L} M_{k}\right), t_{k}^{(0)}<\infty\right]
\end{aligned}
$$

holds. Performing summation on $n$ one has that there exists $\mathfrak{c}>0$ so that

$$
\begin{aligned}
& 2 \sum_{n \geq L^{2}} \bar{E}_{0}\left[\exp \left(c \kappa^{L} X_{S_{k+1}} \cdot l\right), t_{k}^{(n)}<\infty, B_{n, k}, A_{n, k}\right] \\
& \leq \mathfrak{c} \kappa^{L} L^{2} \frac{1}{\exp \left(-c|l|_{\infty} \kappa^{L} L^{2}\right)-\left(1-\widetilde{c} L^{2} \kappa^{L}\right)} \bar{E}_{0}\left[\exp \left(c \kappa^{L} M_{k}\right), t_{k}^{(0)}<\infty\right] .
\end{aligned}
$$

It follows that for some small enough constant $c>0$, there exists $c>0$ such that

$$
\begin{aligned}
& \sum_{n \geq L^{2}} \bar{E}_{0}\left[\exp \left(c \kappa^{L} X_{S_{k+1}} \cdot l\right), t_{k}^{(n)}<\infty, B_{n, k}, A_{n, k}\right] \\
& \leq \mathrm{c} \bar{E}_{0}\left[\exp \left(c \kappa^{L} M_{k}\right), t_{k}^{0}<\infty\right] \leq \mathrm{c} \bar{E}_{0}\left[\exp \left(c \kappa^{L} M_{k}\right), R_{k}<\infty\right] \\
& =\mathrm{c} \bar{E}\left[\exp \left(c \kappa^{L} X_{S_{k}} \cdot l\right), S_{k}<\infty, \exp \left(\kappa^{L}\left(M_{k}-l \cdot X_{S_{k}}\right)\right), D^{\prime} \circ \theta_{S_{k}}<\infty\right] .
\end{aligned}
$$

Using the Markov property and the product structure of the probability measure $Q$, we have

$$
\begin{array}{r}
\bar{E}_{0}\left[\exp \left(c \kappa^{L} X_{S_{k}}\right), S_{k}<\infty, \exp \left(\kappa^{L}\left(M_{k}-l \cdot X_{S_{k}}\right)\right), D^{\prime} \circ \theta_{S_{k}}<\infty\right] \\
=\sum_{x \in \mathbb{Z}^{d}, n \in \mathbb{N}} \mathbb{E}\left[E_{Q \otimes P_{\varepsilon, \omega}^{0}}\left[\exp \left(c \kappa^{L} x \cdot l\right), S_{k}=n, X_{n}=x\right]\right. \\
\left.\times E_{Q \otimes P_{\theta_{n}, \theta_{x} \omega}}\left[\exp \left(c \kappa^{L} \bar{M}\right), D^{\prime}<\infty\right]\right],
\end{array}
$$

provided we define:

$$
\bar{M}=\sup _{0 \leq n \leq D^{\prime}}\left\{\left(X_{n}-X_{0}\right) \cdot l\right\}
$$

imsart-aop ver. 2014/10/16 file: trenormrev.tex date: November 19, 2018 
At this point we can apply the same sort of procedure as the one developed to get the rightmost expression in (4.4). More precisely, the last expression in (4.9) can be bounded from above by means of the following sequence of steps (recall definition (3.1), together with sets $H$ and $\Lambda$, introduced after $(4.4))$ :

$$
\begin{aligned}
& \sum_{x \in \mathbb{Z}^{d}, n \in \mathbb{N}} \mathbb{E}\left[E_{Q \otimes P_{\varepsilon, \omega}^{0}}\left[\exp \left(c \kappa^{L} x \cdot l\right), S_{k}=n, X_{n}=x\right]\right. \\
\times & \left.E_{Q \otimes P_{\theta_{n} \varepsilon, \theta_{x} \omega}}\left[\exp \left(c \kappa^{L} \bar{M}\right), D^{\prime}<\infty\right]\right] \\
= & \sum_{x \in \mathbb{Z}^{d}, n \in \mathbb{N}} \mathbb{E}\left[E_{Q \otimes P_{\varepsilon, \omega}^{0}}\left[\exp \left(c \kappa^{L} x \cdot l\right), S_{k}=n, X_{n}=x\right]\right. \\
\times & \left.\mathbb{E}\left[E_{Q \otimes P_{\theta_{n} \varepsilon, \theta_{x} \omega}}\left[\exp \left(c \kappa^{L} \bar{M}\right), D^{\prime}<\infty\right] \mid \mathfrak{F}_{x, L}\right]\right] \\
\leq & \sum_{x \in \mathbb{Z}^{d}, n \in \mathbb{N}} \mathbb{E}\left[E_{Q \otimes P_{\varepsilon, \omega}^{0}}\left[\exp \left(c \kappa^{L} x \cdot l\right), S_{k}=n, X_{n}=x\right]\right. \\
\times & \left.\exp \left(C \sum_{x \in \partial^{r}\left(H^{c}\right), y \in \partial^{r}\left(\Lambda^{c}\right)} e^{-g|x-y|_{1}}\right) \times \bar{E}_{0}\left[\exp \left(c \kappa^{L} \bar{M}\right), D^{\prime}<\infty\right]\right] \\
\leq & 2 \bar{E}_{0}\left[\exp \left(c \kappa^{L} X_{S_{k}} \cdot l\right), S_{k}<\infty\right] \times \bar{E}_{0}\left[\exp \left(c \kappa^{L} \bar{M}\right), D^{\prime}<\infty\right] .
\end{aligned}
$$

Thus an induction argument makes us conclude that for a suitable constant c $>0$,

$$
\begin{aligned}
& \bar{E}_{0}\left[\exp \left(c \kappa^{L} X_{S_{k+1}} \cdot l\right), S_{k+1}<\infty\right] \\
& \leq\left(\bar{E}_{0}\left[c \exp \left(c \kappa^{L} \bar{M}\right), D^{\prime}<\infty\right]\right)^{k} \times \bar{E}_{0}\left[\exp \left(c \kappa^{L} X_{S_{1}} \cdot l\right), S_{1}<\infty\right] .
\end{aligned}
$$

On the other hand, for $k=0$, the inequality (4.7) is still being true. As a consequence, one can obtain the same upper bound as in the rightmost expression of (4.8) when $k=0$ (which implies in turn that $M_{0}=0$ ). Hence as a result,

$$
\bar{E}_{0}\left[\exp \left(c \kappa^{L} X_{S_{k}} \cdot l\right), S_{k}<\infty\right] \leq\left(E\left[\mathbf{c} \exp \left(c \kappa^{L} \bar{M}\right), D^{\prime}<\infty\right]\right)^{k}
$$

holds.

The following auxiliary result will finish the proof.

Lemma 4.2 (under $\left.(\mathbf{T})_{\ell}\right)$. There exist constants $c_{4}, c_{5}>0$, such that

$$
E_{0}\left[\exp \left(c_{4} \bar{M}\right), D^{\prime}<\infty\right]<c_{5}
$$


Proof. We observe that replacing $c$ by $c /|l|_{2}$ below, it will be sufficient to prove that for some $c>0$, there exists finite $c^{\prime}>0$ such that

$$
E_{0}\left[\exp \left(c M^{\prime}\right), D^{\prime}<\infty\right]<c^{\prime},
$$

where as a matter of definition, we have denoted by:

$$
M^{\prime}=\sup _{0 \leq n \leq D^{\prime}}\left\{\left(X_{n}-X_{0}\right) \cdot \ell\right\}(\text { cf. }(2.9)) .
$$

Notice that

$$
\begin{aligned}
& E_{0}\left[\exp \left(c M^{\prime}\right), D^{\prime}<\infty\right] \leq e^{c} P_{0}\left[D^{\prime}<\infty\right]+ \\
& \sum_{m \geq 0} \exp \left(c 2^{m+1}\right) P_{0}\left[2^{m} \leq M^{\prime}<2^{m+1}, D^{\prime}<\infty\right] .
\end{aligned}
$$

As a consequence of the previous decomposition inequality, it suffices to obtain an appropriate upper bound for large $m$ of the probability:

$$
P_{0}\left[2^{m} \leq M^{\prime}<2^{m+1}, D^{\prime}<\infty\right]
$$

To this end, it will be convenient to introduce the following stopping time for the canonical filtration of the walk:

$$
D^{\prime}(0)=\inf \left\{n \geq 0: X_{n} \notin C(0, l, \zeta)\right\}
$$

Plainly, using the notation of (1.4)-(2.2) one has the inequality:

$$
\begin{aligned}
& P_{0}\left[2^{m} \leq M^{\prime}<2^{m+1}, D^{\prime}<\infty\right] \\
& \leq P_{0}\left[T_{2^{m}}^{\ell} \leq D^{\prime}<\infty, T_{2^{m+1}}^{\ell} \circ \theta_{T_{2^{m}}^{\ell}}>D^{\prime}(0) \circ \theta_{T_{2^{m}}^{\ell}}\right] \\
& \leq P_{0}\left[X_{T_{2^{m}}^{\ell}} \notin \partial^{+} B_{2^{m}, \mathfrak{r}^{m}, \ell}(0), T_{2^{m}}^{\ell} \leq D^{\prime}<\infty\right] \\
& +P_{0}\left[X_{T_{2^{m}}^{\ell}} \in \partial^{+} B_{2^{m}, 2^{m}, \ell}(0), T_{2^{m+1}}^{\ell} \circ \theta_{T_{2^{m}}^{\ell}}>D^{\prime}(0) \circ \theta_{T_{2^{m}}^{\ell}}\right] .
\end{aligned}
$$

Notice that on the event of the first probability on the rightmost expression in $(4.14), P_{0}$-a.s. one has

$$
X_{T_{B_{2}{ }^{m}, \mathbf{r}^{m}, \ell}(0)} \notin \partial^{+} B_{2^{m}, \mathfrak{r} 2^{m}, \ell}(0) .
$$

Therefore, condition $(\mathbf{T})_{\ell}$ implies that for large $m$,

$$
\begin{array}{r}
P_{0}\left[X_{T_{2^{m}}^{\ell}} \notin \partial^{+} B_{2^{m},\left(\frac{2}{\varepsilon}\right) 2^{m}, \ell}(0), T_{2^{m}}^{\ell} \leq D^{\prime}<\infty\right] \\
\leq \exp \left(-\mathfrak{c} 2^{m}\right)
\end{array}
$$


for some suitable positive constant $\mathfrak{c}$. As for the second term on the rightmost expression of (4.14), for $m \in \mathbb{N}$ we introduce the boundary box $F_{m}$ via:

$$
F_{m}=\partial^{+} B_{2^{m}, \mathfrak{r} 2^{m}, \ell}(0) \text {. }
$$

Applying the strong Markov property we find that

$$
\begin{aligned}
& P_{0}\left[X_{T_{2 m}^{\ell}} \in \partial^{+} B_{2^{m}, \mathfrak{r}^{m}, \ell}(0), T_{2^{m+1}}^{\ell} \circ \theta_{T_{2^{m}}^{\ell}}>D^{\prime}(0) \circ \theta_{T_{2^{m}}^{\ell}}\right] \\
& \leq \sum_{y \in F_{m}} P_{y}\left[T_{2^{m+1}}^{\ell}>D^{\prime}(0)\right] .
\end{aligned}
$$

In order to estimate the rightmost probability entering in (4.17), we will bound from below the probability of its complementary event as follows. Introducing for $x \in \mathbb{Z}^{d}$, the set:

$$
B_{x}=B_{2^{m-1}, \mathfrak{r} 2^{m-1}, \ell}(x),
$$

we note that under the assumption (4.1) we have

$$
\mathfrak{r}\left(2^{m}+2^{m-1}\right) \leq \tan \left(\frac{\pi}{2}-\arccos (\zeta)\right) 2^{m-1},
$$

which implies that the boxes $B_{y}$ and $B_{z}$, where $y \in F_{m}$ and $z \in \partial^{+} B_{y}$, are both inside of the cone $C(0, l, \zeta)$ (see Figure 1 below).

Observe that for $y \in F_{m}$, one has the following lower bound:

$$
\begin{aligned}
& P_{y}\left[T_{2^{m+1}}^{\ell}<D^{\prime}(0)\right] \\
& \geq \sum_{z \in \partial^{+} B_{y}} \mathbb{E}\left[P_{y, \omega}\left[X_{T_{B y}} \in \partial^{+} B_{y}, X_{T_{B y}}=z,\left(X_{T_{B z}} \in \partial^{+} B_{z}\right) \circ \theta_{T_{B y}}\right]\right] .
\end{aligned}
$$

To estimate the right-hand side of the above inequality, it will be convenient to introduce for $m \in \mathbb{N}$, the second boundary set $\bar{F}_{m}$ as

$$
\bar{F}_{m}:=\partial\left[\cup_{y \in F_{m}} B_{y}\right] \cap R\left(\left[2^{m-1}+2^{m}, \infty\right) \times \mathbb{R}^{d-1}\right),
$$

and in turn for that given set $\bar{F}_{m}$ we introduce the good environment event $G_{\bar{F}_{m}}$ by

$$
\begin{aligned}
G_{\bar{F}_{m}}:= & \left\{\omega \in \Omega: P_{z, \omega}\left[X_{T_{B z}} \in \partial^{+} B_{z}\right]>\right. \\
& \left.1-\exp \left(-\mathrm{c} 2^{(m-1)}\right), \text { for all } z \in \bar{F}_{m}\right\},
\end{aligned}
$$

imsart-aop ver. 2014/10/16 file: trenormrev.tex date: November 19, 2018 


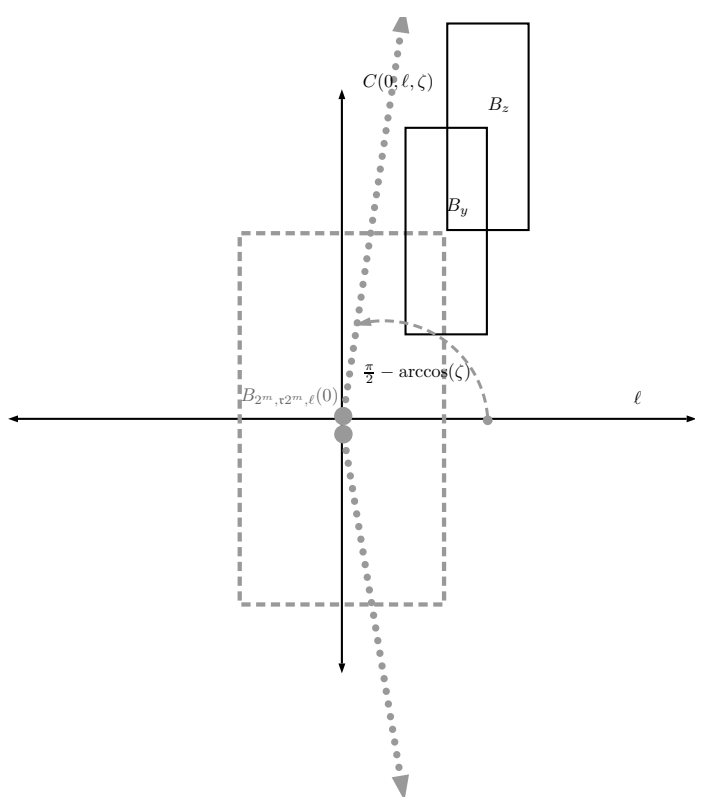

FIG 1. Boxes $B_{y}$ and $B_{z}$ are inside of $C(0, l, \zeta)$.

where the constant $\mathrm{c}>0$ will be chosen below. Using the strong Markov property, we can now bound from below the right-hand side of inequality (4.19) by

$$
\left(1-\exp \left(-c 2^{(m-1)}\right)\right)\left(P_{y}\left[X_{T_{B y}} \in \partial^{+} B_{y}\right]-P_{y}\left[\left(G_{\bar{F}_{m}}\right)^{c}\right]\right)
$$

where for an event $E$, we denote by $(E)^{c}$ its complementary event.

Furthermore, using stationarity under the probability measure $\mathbb{P}$ and condition $(\mathbf{T})_{\ell}$, for $x \in \mathbb{R}^{d}$ and large $m$ one has

$$
\begin{aligned}
P_{x}\left[X_{T_{B x}} \notin \partial^{+} B_{x}\right]= & P_{0}\left[X_{T_{B_{0}}} \notin \partial^{+} B_{0}\right] \\
& \leq \exp \left(-\mathfrak{w} 2^{m-1}\right),
\end{aligned}
$$

for a suitable $\mathfrak{w}>0$.

We thus see that (4.20) is greater than

$$
\left(1-\exp \left(-\mathrm{c} 2^{(m-1)}\right)\right)\left(1-\exp \left(-\mathfrak{w} 2^{m-1}\right)-P_{y}\left[\left(G_{\bar{F}_{m}}\right)^{c}\right]\right)
$$


Taking $\mathrm{c}=\mathfrak{w} / 2$, in virtue of (4.21) and Chevyshev's inequality we find that

$$
\begin{array}{r}
P_{y}\left[\left(G_{\bar{F}_{m}}\right)^{c}\right] \\
\leq\left|\bar{F}_{m}\right| \exp \left(\mathrm{c} 2^{(m-1)}\right) \sup _{x \in \bar{F}_{m}} P_{x}\left[X_{T_{B x}} \notin \partial^{+} B_{x}\right] \leq \exp \left(-\mathfrak{t} 2^{m-2}\right),
\end{array}
$$

for a suitable $\mathfrak{t}>0$, where we have used for $m \in \mathbb{N}$ the coarse estimate:

$$
\max \left(\left|\bar{F}_{m}\right|,\left|F_{m}\right|\right) \leq\left(6 \mathfrak{r} 2^{m}\right)^{d-1} .
$$

Consequently, for large $m$ we can find a further positive constant $\widetilde{c}$ such that:

$$
P_{y}\left[T_{2^{m+1}}^{\ell} \leq D^{\prime}(0)\right] \geq 1-\exp \left(-\widetilde{c} 2^{m}\right)
$$

for all $y \in \bar{F}_{m}$.

In view of (4.16), (4.17) and (4.24), the claim (4.12) follows.

As it was mentioned the assertion in (4.2) follows from (4.12) and $P_{0}\left[D^{\prime}=\right.$ $\infty]>0$, with the help of estimate (4.11).

We are now ready to spell out some consequences of the previous proposition. We first define the random variable $Y$ as

$$
Y=\sup _{0 \leq n \leq \tau_{1}}\left|X_{n}\right|_{2}
$$

We can prove the following reinforcement to Theorem 4.1:

Corollary 4.3 (under $\left.(\mathbf{T})_{\ell}\right)$. Assume either: $(\boldsymbol{S} \boldsymbol{M})_{C, g}$ or $(\boldsymbol{S M G})_{C, g}$. Then there exist positive constants $c_{6}, c_{7}$ and $L_{0}$ such that

$$
\bar{E}_{0}\left[e^{c_{6} \kappa^{L} Y}\right] \leq c_{7}
$$

provided that $L \geq L_{0}, L \in|l|_{1} \mathbb{N}$.

Proof. Using item (ii) of Lemma 2.2, notice that for large $u$,

$$
\begin{aligned}
& \quad \bar{P}_{0}[Y \geq u]=\bar{P}_{0}\left[\sup _{0 \leq n \leq \tau_{1}}\left|X_{n}\right|_{2} \geq u\right] \\
& \leq \bar{P}_{0}\left[T_{\Delta_{\frac{u}{2}}}<\tau_{1}\right] \\
& \leq \bar{P}_{0}\left[X_{\tau_{1}} \cdot l \geq \frac{u}{2 \hat{r}}\right]+\bar{P}_{0}\left[X_{\tau_{1}} \cdot l<\frac{u}{2 \hat{r}}, T_{\Delta_{\frac{u}{2 \hat{r}}}}<\tau_{1}\right] \\
& \quad \leq \exp \left(-\kappa^{L} c_{2} \frac{u}{2 r}\right) \bar{E}_{0}\left[\exp \left(c_{2} \kappa^{L} X_{\tau_{1}} \cdot l\right)\right]+P_{0}\left[X_{T_{\Delta_{\frac{u}{2}}}} \notin \partial^{+} \Delta_{\frac{w}{2 \hat{r}}}\right] \\
& \text { imsart-aop ver. 2014/10/16 file: trenormrev.tex date: November 19, } 2018
\end{aligned}
$$


where in the last step we have used that by definition $X_{m} \cdot l<X_{\tau_{1}} \cdot l$, when $0 \leq m<\tau_{1}$. Keeping in mind the layer cake decomposition (cf. [Ru87], Chapter 8, Theorem 8.16), the claim of the corollary follows after applying condition $(\mathbf{T})_{\ell}$ and Proposition 4.1.

In order to state the next proposition it will be useful to fix some further notation. For $L \in|l|_{1} \mathbb{N}$, we introduce the approximate asymptotic direction denoted as $\hat{v}_{L} \in \mathbb{S}^{d-1}$ and given by

$$
\hat{v}_{L}:=\frac{\bar{E}_{0}\left[X_{\tau_{1}} \mid D^{\prime}=\infty\right]}{\left|\bar{E}_{0}\left[X_{\tau_{1}} \mid D^{\prime}=\infty\right]\right|} .
$$

which a priori depends on $L$, however when there is not risk of confusion, we shall drop it.

As explained in [GR17] Proposition 7.2. page 34, one has:

Proposition 4.4. There exist positive constants $k_{1}$ and $k_{2}$ (not depending on $L$ ) such that for any $L \in|l|_{1} \mathbb{N}$,

$$
\bar{E}_{0}\left[\left(\kappa^{L} X_{\tau_{1}} \cdot l\right) \mid D^{\prime}=\infty\right] \geq k_{1} \quad \text { and } \quad\left|\bar{E}_{0}\left[\left(\kappa^{L} X_{\tau_{1}}\right) \mid D^{\prime}=\infty\right]\right|_{2} \geq k_{2} .
$$

Thus, the upper bounds obtained in this sections are sharps. Proposition 4.4 will be useful to prove Theorem 6.3 in Section 6 .

We continue with the definition for $t \in \mathbb{R}$ of the random variable

$$
M_{t}:=\sup \left\{n \geq 0: X_{n} \cdot l \leq t\right\},
$$

this is the last visit to the half space $H=\{z: z \cdot l \leq t\}$. We also define the projector operator $\Pi=\Pi_{\hat{v}}: \mathbb{R}^{d} \rightarrow \mathbb{R}^{d}$ onto the orthogonal space to $\hat{v}_{L}$, so that for $z \in \mathbb{R}^{d}$

$$
\Pi(z)=z-(z \cdot \hat{v}) \hat{v} .
$$

The next proposition will be fundamental to apply renormalization arguments in order to obtain annealed estimates of atypical quenched escapes for the walk.

Proposition 4.5 (under $(\mathbf{T})_{\ell}$, see (2.9)). Let $C, g>0$ and assume either: $(\boldsymbol{S M})_{C, g}$ or $(\boldsymbol{S M G})_{C, g}$ and (1.5). Let $\gamma \in(5 / 9,1)$ and $\rho>0$. Then there exists $c_{8}=c_{8}(d, \rho, \kappa, l)>0$, so that for large $u$ one has that

$$
P_{0}\left[\sup _{0 \leq n \leq M_{u}}\left|\Pi_{\hat{v}}\left(X_{n}\right)\right| \geq \rho u^{\gamma}\right] \leq \exp \left(-c_{8} u^{\frac{9}{4} \gamma-\frac{5}{4}}\right),
$$

imsart-aop ver. 2014/10/16 file: trenormrev.tex date: November 19, 2018 
with the notation as in (4.29) and $\hat{v}=\hat{v}_{L}$ is the vector defined by (4.28), where for a fixed number $t \in(1 / 2,1)$ with

$$
g t>18 \log \left(\frac{1}{\kappa}\right)
$$

$L$ is the least integer in $|l|_{1} \mathbb{N}$, such that:

$$
\exp (-g t L) \leq u^{2 \gamma-2+\frac{\gamma-1}{4}}
$$

Proof. Fix $\gamma \in(5 / 9,1), t$ as in (4.31) and consider a large enough $u$ so that the least integer $L \in|l|_{1} \mathbb{N}$ satisfying

$$
\exp (-g t L) \leq u^{2 \gamma-2+\frac{\gamma-1}{4}}
$$

is also satisfying the following requirements:

$$
\begin{aligned}
& L \geq L_{0}, \\
& L \geq \frac{6\left(2 c_{7}+1\right)|l|_{1} e^{\frac{g|l|_{1}}{36}}}{|l|_{2} \rho c_{6}} \text { and } \\
& u^{2(\gamma-1)+\left(\frac{\gamma-1}{4}\right)} \leq \frac{1}{2} .
\end{aligned}
$$

Above, constant $L_{0}$ is as in the statement of Corollary 4.3. For the rest of the proof, we will drop the prescribed $L$ defined by (4.32) and satisfying (4.33)-(4.35)) from the notation, to set for instance: $\hat{v}=\hat{v}_{L}, \tau_{1}=\tau_{1}^{(L)}$, and so on. Furthermore, notice that it is sufficient to prove an analogue inequality to (4.30), replacing $\Pi_{\hat{v}}\left(X_{n}\right)$ by $X_{n} \cdot w$, where $w \in \mathbb{S}^{d-1}$ with $w \cdot \hat{v}=0$. Therefore, we will prove the proposition under this convention and we introduce for $n \in \mathbb{N}$ the random variable $K_{n}$, via

$$
K_{n}=\sup \left\{k \geq 0: \tau_{k} \leq n\right\} \quad\left(\text { set } \tau_{0}=0 .\right)
$$

Since $\bar{P}_{0}$-a.s. one has for $m \leq \tau_{1} \leq m^{\prime}$ :

$$
X_{m} \cdot l \leq X_{\tau_{1}} \cdot l \leq X_{m^{\prime}} \cdot l \text { and } X_{\tau_{1}} \cdot l \geq L \frac{|l|_{2}}{|l|_{1}}
$$

it follows that $\bar{P}_{0}$-a.s.

$$
0 \leq n \leq M_{u} \Rightarrow K_{n} \leq \frac{|l|_{1}}{|l|_{2} L} u
$$


Hence, for $n \in\left[0, M_{u}\right]$ and $K_{n}$ as above, we have (recall the notation in display (4.25))

$$
X_{n} \cdot w=\left(X_{n}-X_{\tau_{K_{n}}}\right) \cdot w+X_{\tau_{K_{n}}} \cdot w \leq Y \circ \theta_{K_{n}}+X_{\tau_{K_{n}}} \cdot w,
$$

and consequently for $\rho>0$ we get the inequality:

$$
\begin{array}{r}
P_{0}\left[\sup _{0 \leq n \leq M_{u}} X_{n} \cdot w \geq \rho u^{\gamma}\right] \leq \sum_{0 \leq k \leq \frac{|l|_{1}}{\mid l l_{2} L} u} \bar{P}_{0}\left[Y \circ \theta_{\tau_{k}} \geq \frac{\rho}{3} u^{\gamma}\right] \\
+\bar{P}_{0}\left[X_{\tau_{1}} \cdot w \geq \frac{\rho}{3} u^{\gamma}\right]+\sum_{2 \leq k \leq \frac{|l|_{1}}{|l|_{2} L} u} \bar{P}_{0}\left[\left(X_{\tau_{k}}-X_{\tau_{1}}\right) \cdot w \geq \frac{\rho}{3} u^{\gamma}\right] .
\end{array}
$$

Let $\lambda \in\left[0, c_{6} \kappa^{L}\right]$ and observe that an application of Chernoff bound leads us to

$$
\begin{aligned}
& P_{0}\left[\sup _{0 \leq n \leq M_{u}} X_{n} \cdot w \geq \rho u^{\gamma}\right] \leq \exp \left(-\lambda \frac{\rho}{3} u^{\gamma}\right)\left(\sum_{0 \leq k \leq \frac{|l|_{1}}{|l|_{2} L} u} \bar{E}_{0}\left[\exp \left(\lambda Y \circ \theta_{\tau_{k}}\right)\right]\right. \\
& \left.(4.37)+\bar{E}_{0}\left[\exp \left(\lambda X_{\tau_{1}} \cdot w\right)\right]+\sum_{2 \leq k \leq \frac{|l|_{1}}{\mid l l_{2} L} u} \bar{E}_{0}\left[\exp \left(\lambda\left(X_{\tau_{k}}-X_{\tau_{1}}\right) \cdot w\right)\right]\right)
\end{aligned}
$$

Let us now perform some computations required to estimate the expectations entering in the last expression above. We first observe that for integer $k \geq 0$

$$
\begin{aligned}
\bar{E}_{0}\left[\exp \left(\lambda Y \circ \theta_{\tau_{k}}\right)\right] & =\sum_{k \geq 1, n \in \mathbb{N}, x \in \mathbb{Z}^{d}} \mathbb{E}\left[E_{Q \otimes P_{\varepsilon, \omega}^{0}}\left[\mathbb{1}_{S_{k}=n, X_{S_{k}}=x}\right]\right. \\
& \left.\times E_{Q \otimes P_{\theta_{n \varepsilon}, \theta_{x} \omega}}\left[\exp (\lambda Y), D^{\prime}=\infty\right]\right] \\
& =\sum_{k \geq 1, n \in \mathbb{N}, x \in \mathbb{Z}^{d}} \mathbb{E}\left[E_{Q \otimes P_{\varepsilon, \omega}^{0}}\left[\mathbb{1}_{S_{k}=n, X_{S_{k}}=x}\right]\right. \\
& \left.\times \mathbb{E}\left[E_{Q \otimes P_{\theta_{n}, \theta_{x} \omega}^{0}}\left[\exp (\lambda Y), D^{\prime}=\infty\right] \mid \mathfrak{F}_{x, L}\right]\right] .
\end{aligned}
$$

Using the proof of the Proposition 3.1, it is easy to see that for the nonnegative random variable $\lambda Y$, the inequality

$$
\begin{aligned}
& \mathbb{E}\left[E_{Q \otimes P_{\theta_{n}, \theta_{x} \omega}^{0}}\left[\exp (\lambda Y) \mid D^{\prime}=\infty\right] \mid \mathfrak{F}_{x, L}\right] \\
& \leq \exp \left(e^{-g t L}\right) \bar{E}_{0}\left[\exp (\lambda Y) \mid D^{\prime}=\infty\right]
\end{aligned}
$$

imsart-aop ver. 2014/10/16 file: trenormrev.tex date: November 19, 2018 
holds.

Therefore, as a result we get for integer $k \geq 0$ the estimate

$$
\begin{aligned}
& \max \left\{\bar{E}_{0}\left[\exp \left(\lambda Y \circ \theta_{\tau_{k}}\right)\right], \bar{E}_{0}\left[\exp \left(\lambda X_{\tau_{1}} \cdot w\right)\right]\right\} \\
& =\bar{E}_{0}\left[\exp \left(\lambda Y \circ \theta_{\tau_{k}}\right)\right] \leq 2 \bar{E}_{0}\left[\exp (\lambda Y) \mid D^{\prime}=\infty\right] .
\end{aligned}
$$

On the other hand, quit a similar procedure but now using the complete statement of Proposition 3.1 along successive conditioning, allows us to conclude that for $k \in\left[2,|l|_{1} u /|l|_{2} L\right]$ one has:

$$
\begin{aligned}
\bar{E}_{0}\left[\exp \left(\lambda\left(X_{\tau_{k}}-X_{\tau_{1}}\right) \cdot w\right)\right] & =\bar{E}_{0}\left[\exp \left(\lambda \sum_{j=2}^{k}\left(X_{\tau_{j}}-X_{\tau_{j-1}}\right) \cdot w\right)\right] \\
& \leq\left(\exp \left(e^{-g t L}\right) \bar{E}_{0}\left[\exp \left(\lambda X_{\tau_{1}} \cdot w\right) \mid D^{\prime}=\infty\right]\right)^{k-1} \\
& \stackrel{(4.36)}{\leq}\left(\exp \left(e^{-g t L}\right) \bar{E}_{0}\left[\exp \left(\lambda X_{\tau_{1}} \cdot w\right) \mid D^{\prime}=\infty\right]\right)^{\frac{|l|_{1} u}{\mid l l_{2} L}} .
\end{aligned}
$$

Define now for $|\lambda| \leq \kappa^{L} c_{6}$, the function

$$
H(\lambda):=\bar{E}_{0}\left[\exp \left\{\lambda X_{\tau_{1}} \cdot w\right\} \mid D^{\prime}=\infty\right] .
$$

Taking $\lambda=\varrho u^{\frac{5}{4}(\gamma-1)}$ for a positive constant $\varrho$ chosen so that

$$
\frac{c_{6}}{2} e^{-g \frac{|l|_{1}}{36}}<\varrho<c_{6} e^{-g \frac{\mid l l_{1}}{36}}
$$

holds, from the very definition of $L$ in (4.32), we obtain

$$
\varrho u^{\frac{\gamma-1}{8}}<\varrho e^{-\frac{g t\left(L-|l|_{1}\right)}{18}} \leq c_{6} e^{-\log (1 / \kappa) L} .
$$

We observe that, for our choice of $\lambda, w \perp \hat{v}$, Proposition 4.1 and Lebesgue's dominated convergence theorem, one has:

$$
\begin{aligned}
H(\lambda) & =\bar{E}_{0}\left[1+\lambda X_{\tau_{1}} \cdot w+\frac{\lambda^{2}}{2 !}\left(X_{\tau_{1}} \cdot w\right)^{2}+\frac{\lambda^{3}}{3 !}\left(X_{\tau_{1}} \cdot w\right)^{2}+\ldots \mid D^{\prime}=\infty\right] \\
& \leq \bar{E}_{0}\left[1+\varrho u^{\gamma-1+\left(\frac{\gamma-1}{4}\right)} X_{\tau_{1}} \cdot w+u^{2(\gamma-1)+\left(\frac{\gamma-1}{4}\right)} \frac{\left(\varrho u^{\frac{\gamma-1}{8}} X_{\tau_{1}} \cdot w\right)^{2}}{2 !}\right. \\
& \left.+u^{3(\gamma-1)+\left(\frac{2(\gamma-1)}{4}\right)} \frac{\left(\varrho u^{\frac{\gamma-1}{8}} X_{\tau_{1}} \cdot w\right)^{3}}{3 !}+\ldots \mid D^{\prime}=\infty\right] \\
& \leq 1+c_{7} u^{2(\gamma-1)+\left(\frac{\gamma-1}{4}\right)} \sum_{j=0}^{\infty} u^{j\left((\gamma-1)+\left(\frac{\gamma-1}{4}\right)\right)} \\
& \leq .35 \\
& \leq 1+2 c_{7} u^{2(\gamma-1)+\left(\frac{\gamma-1}{4}\right)} \leq e^{2 c_{7} u^{2(\gamma-1)+\left(\frac{\gamma-1}{4}\right)} .}
\end{aligned}
$$


Consequently, once again since (4.32) and requirement (4.33) we have that

$$
\begin{aligned}
(4.39) & =\left(\exp \left(e^{-g t L}\right) \exp \left(\log \left(\bar{E}_{0}\left[\exp \left(\lambda X_{\tau_{1}} \cdot w\right) \mid D^{\prime}=\infty\right]\right)\right)\right)^{\frac{|l|_{1} u}{|l|_{2} L}} \\
& \leq \exp \left(\left(2 c_{7}+1\right) u^{2 \gamma-2+\left(\frac{\gamma-1}{4}\right)} \times\left(\frac{|l|_{1} u}{|l|_{2} L}\right)\right) .
\end{aligned}
$$

Inserting estimates (4.38) and (4.41) into (4.37) the assertion of the proposition follows since assumption (4.34).

REMARK 4.6. Let us sketch the proof for finite dependent random environments. Taking $L$ large enough with respect to the dependence of the environment we get to the rightmost expression in (4.39) without factor $\exp \left(e^{-g t L}\right)$. Then, it is direct to see that i.i.d. renormalization techniques can be applied in this case without the help of assumption (1.5). Here, the crucial point is that there exists a finite $L$ such that $\tau_{1}^{(L)}$ is in fact a regeneration time.

5. Estimates for the Regeneration Time Tails. The main objective of this section will be to obtain an upper bound for the probability $\bar{P}_{0}\left[\tau_{1} \geq u\right]$ when $u$ is large and independent of $L$. Let $C, g>0$, throughout the complete section we shall assume condition $(\mathbf{T})_{\ell}$, where $\ell \in \mathbb{S}^{d-1}$ satisfies $(2.9)$, and either: $(\mathbf{S M})_{C, g}$ or $(\mathbf{S M G})_{C, g}$. We first prove a basic lemma in the spirit of [Sz00], Lemma 1.3. It is convenient to fix a rotation $R$ on $\mathbb{R}^{d}$, with

$$
R\left(e_{1}\right)=\frac{l}{|l|_{2}}=\ell
$$

Introducing for $M>0$, the hypercube

$$
C_{M}:=B_{M, \mathfrak{r} M, \ell}(0)
$$

We have

Lemma 5.1. There exist $c_{9}>0$ and $L_{0}>0, L_{0} \in|l|_{1} \mathbb{N}$ such that for any function $M: \mathbb{R}^{+} \rightarrow \mathbb{R}^{+}$, with $\lim _{u \rightarrow \infty} M(u)=\infty$ one has that for large $u$,

$$
\bar{P}_{0}\left[\tau_{1}>u\right] \leq P_{0}\left[T_{C_{M(u)}}=T_{M(u)}^{l}>u\right]+e^{-c_{9} \kappa^{L} M(u)}
$$

for each $L \in|l|_{1} \mathbb{N}, L \geq L_{0}$. 
Proof. Let us start with the inequality

$$
\begin{gathered}
\bar{P}_{0}\left[\tau_{1}>u\right] \leq \bar{P}_{0}\left[\tau_{1}>u, X_{\tau_{1}} \cdot l \leq|l|_{2} M(u)\right]+\bar{P}_{0}\left[X_{\tau_{1}} \cdot l>|l|_{2} M(u)\right] \\
\leq \bar{P}_{0}\left[\tau_{1}>u, X_{\tau_{1}} \cdot l\right. \\
\left.\leq|l|_{2} M(u)\right]+e^{-c_{1} \kappa^{L}|l|_{2} M(u)} \bar{E}_{0}\left[\exp \left(c_{1} \kappa^{L} X_{\tau_{1}} \cdot l\right)\right] \\
\leq \bar{P}_{0}\left[\tau_{1}>u, X_{\tau_{1}} \cdot l \leq|l|_{2} M(u)\right]+e^{-\frac{c_{1} \mid l l_{2} \kappa^{L} M(u)}{2}} .
\end{gathered}
$$

It is then sufficient to estimate the probability

$$
\bar{P}_{0}\left[\tau_{1}>u, X_{\tau_{1}} \cdot l \leq|l|_{2} M(u)\right] .
$$

From the definition of time $\tau_{1}$, one has that $\tau_{1}=T_{X_{\tau_{1}} \cdot l}^{l}$. Hence, we find that

$$
\bar{P}_{0}\left[\tau_{1}>u, X_{\tau_{1}} \cdot l \leq M(u)\right] \leq P_{0}\left[T_{|l|_{2} M(u)}^{l}>u\right] \stackrel{(1.4)}{=} P_{0}\left[T_{M(u)}^{\ell}>u\right] .
$$

We first proceed to consider the following decomposition inequality

$$
P_{0}\left[T_{M(u)}^{\ell}>u\right] \leq P_{0}\left[T_{C_{M(u)}}=T_{M(u)}^{\ell}>u\right]+P_{0}\left[T_{C_{M(u)}}<T_{M(u)}^{\ell}\right],
$$

for large $u$. Since $(\mathbf{T})_{\ell}$ holds, (see (2.1) and Lemma 2.2)

$$
\begin{aligned}
P_{0}\left[T_{C_{M(u)}}<T_{M(u)}^{l^{\prime}}\right] & \leq P_{0}\left[X_{\left.T_{C_{M(u)}} \notin \partial^{+} C_{M(u)}\right]}\right. \\
& \leq \exp (-\widetilde{c} M(u)),
\end{aligned}
$$

for a suitable constant $\widetilde{c}>0$.

Thus, coming back to (5.2) the required assertion follows from (5.3).

In the next subsection we will present an atypical quenched estimate for mixing environments in the spirit of [Sz00], Proposition 3.1.

5.1. Renormalization. The main objective here is to establish a version of an atypical quenched estimate for mixing random environments in the spirit of Proposition 3.1 in [Sz00] for i.i.d. environments. To this purpose, we first introduce the set

$$
U_{M}=\left\{y \in \mathbb{Z}^{d}:|y \cdot \ell|<M\right\}
$$

for $M>0$. The crucial ingredient to bound from above the tail of $\tau_{1}$ is given below.

Proposition 5.2. For $\beta \in[0,1)$ and $c>0$

$$
\begin{gathered}
\limsup _{M \rightarrow \infty} M^{-\chi} \log \mathbb{P}\left[P_{0, \omega}\left[X_{T_{U_{M}}} \cdot \frac{l}{|l|_{2}} \geq M\right] \leq e^{-c M^{\beta}}\right]<0, \\
\text { where either } \chi=1 \text { or } \chi<d\left(\frac{13}{4} \beta-\frac{9}{4}\right) .
\end{gathered}
$$

imsart-aop ver. 2014/10/16 file: trenormrev.tex date: November 19, 2018 
Proof. By a quit similar argument of [Sz00], page 121, the case $\chi=1$ easily follows from condition $(\mathbf{T})_{\ell}$. We thus only need to consider the case when $\beta \in[0,1)$ is large enough such that

$$
d\left(\frac{13}{4} \beta-\frac{9}{4}\right)>1
$$

The key idea of the proof (cf. [Sz00]) is to construct strategies for the walk ensuring that this starting from $0 \in \mathbb{Z}^{d}$, escapes from $U_{M}$ by the boundary side $\partial^{+} U_{M}:=\partial U_{M} \cap\left\{z \in \mathbb{R}^{d}: z \cdot l /|l|_{2} \geq M\right\}$. Such a construction involves the notion of good and bad boxes for the environment, and they will provide high probability on the event that the walk fulfills the required strategies. In order to introduce the definitions of good and bad boxes, we need some further notation. For $L \geq L_{0}$ with $L \in|l|_{1} \mathbb{N}$, we pick a rotation $\tilde{R}_{L}$ on $\mathbb{R}^{d}$ so that

$$
\left.\tilde{R}_{L}\left(e_{1}\right)=\hat{v}_{L} \text { (we shall only write } \tilde{R} \text {, beacuse we will fix } L \text { below }\right) .
$$

We consider $\gamma \in(5 / 9,1)$ and $t \in(1 / 2,1) \cap \mathbb{Q}$ so that

$$
\operatorname{tg}>18 \log \left(\frac{1}{\kappa}\right) \text {. }
$$

Pick then $M_{0}>2 \sqrt{d}$ large enough, such that if $L$ is the integer satisfying

$$
L=\min \left\{\widehat{L} \in|l|_{1} \mathbb{N}: e^{-g t \widehat{L}} \leq M_{0}^{2 \gamma-2+\left(\frac{\gamma-1}{4}\right)}\right\},
$$

one has that $L \geq L_{0}$ and $L \geq \frac{48|l|_{1}}{\hat{v} \cdot l}$ (which is possible by Proposition 4.4).

Define for $z \in M_{0} \mathbb{Z}^{d}$ ( $M_{0}$ as above), the following blocks:

$$
\begin{aligned}
& \tilde{B}_{1}(z):=\tilde{R}\left(z+\left(0, M_{0}\right)^{d}\right) \cap \mathbb{Z}^{d} \\
& \tilde{B}_{2}(z):=\tilde{R}\left(z+\left(-M_{0}^{\gamma}, M_{0}+M_{0}^{\gamma}\right)^{d}\right) \cap \mathbb{Z}^{d},
\end{aligned}
$$

which are nonempty because $M_{0}>2 \sqrt{d}$. One also defines the boundary positive part of $\tilde{B}_{2}(z)$ via

$$
\partial^{+} \tilde{B}_{2}(z):=\partial \tilde{B}_{2}(z) \cap\left\{y:(y-z) \cdot \tilde{R}\left(e_{1}\right) \geq M_{0}+M_{0}^{\gamma}\right\} .
$$

We then say that site $z \in M_{0} \mathbb{Z}^{d}$ is $M_{0}$-good, if

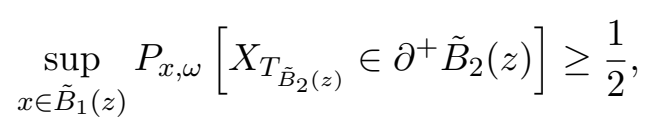

and $M_{0}$-bad otherwise. We have the following upper bound for $M_{0}$-bad blocks:

imsart-aop ver. 2014/10/16 file: trenormrev.tex date: November 19, 2018 
Lemma 5.3. Let $\gamma \in(5 / 9,1)$. Then, one has that

$$
\limsup _{M_{0} \rightarrow \infty} M_{0}^{5 / 4-(9 / 4 \gamma)} \sup _{z \in M_{0} \mathbb{Z}^{d}} \log \mathbb{P}\left[z \text { is } M_{0}-b a d\right]<0 .
$$

ProOf. For $z \in M_{0} \mathbb{Z}^{d}$,

$$
\begin{aligned}
\mathbb{P}\left[z \text { is } M_{0} \text {-bad }\right] & =\mathbb{P}\left[\sup _{x \in \tilde{B}_{1}(z)} P_{x, \omega}\left[X_{T_{\tilde{B}_{2}(z)}} \notin \partial^{+} \tilde{B}_{2}(z)\right]>\frac{1}{2}\right] \\
& \leq 2\left|\tilde{B}_{1}(z)\right| \sup _{x \in \tilde{B}_{1}(z)} P_{x}\left[X_{T_{\tilde{B}_{2}(z)}} \notin \partial^{+} \tilde{B}_{2}(z)\right] .
\end{aligned}
$$

Observe that for $x \in \tilde{B}_{1}(z)$, one has that $\tilde{B}_{2}(z)$ is included in the closed Euclidean ball centered at $x$ of radius $3 \sqrt{d} M_{0}$. Therefore, recalling that $\ell=l /|l|_{2}($ cf. $(2.9))$ one gets $P_{x}$-a.s.

$$
T_{\tilde{B}_{2}(z)} \leq T_{x \cdot l^{\prime}+3 \sqrt{d} M_{0}}^{l^{\prime}} .
$$

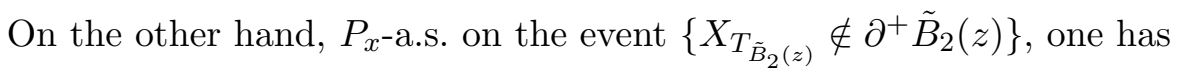

$$
\text { either }\left(X_{T_{\tilde{B}_{2}(z)}}-x\right) \cdot \hat{v} \leq-\frac{M_{0}^{\gamma}}{2} \text { or }\left|\Pi_{\hat{v}}\left(X_{T_{\tilde{B}_{2}(z)}}-x\right)\right|_{2} \geq \frac{M_{0}^{\gamma}}{2} \text {, }
$$

where the notation is as in Proposition 4.5. As a result, one gets

$$
\begin{array}{r}
\mathbb{P}\left[z \text { is } M_{0}-\mathrm{bad}\right] \leq c(d) M_{0}^{d}\left(P_{0}\left[\sup _{0 \leq n \leq T_{3 \sqrt{d} M_{0}}^{\ell}}\left|\Pi_{\hat{v}}\left(X_{n}\right)\right|_{2} \geq \frac{\hat{v} \cdot \ell}{4} M_{0}^{\gamma}\right]\right. \\
\left.+P_{0}\left[\sup _{0 \leq n \leq T_{3 \sqrt{d} M_{0}}^{\ell}}\left|\Pi_{\hat{v}}\left(X_{n}\right)\right|_{2}<\frac{\hat{v} \cdot \ell}{4} M_{0}^{\gamma}, \inf _{0 \leq n \leq T_{3 \sqrt{d} M_{0}}^{\ell}} X_{n} \cdot \hat{v} \leq-\frac{M_{0}^{\gamma}}{2}\right]\right)
\end{array}
$$

$$
\leq c(d) M_{0}^{d}\left(P_{0}\left[\sup _{0 \leq n \leq T_{3 \sqrt{d} M_{0}}^{\ell}}\left|\Pi_{\hat{v}}\left(X_{n}\right)\right|_{2} \geq \frac{\hat{v} \cdot \ell}{4} M_{0}^{\gamma}\right]+P_{0}\left[\widetilde{T}_{-\frac{M_{0}^{\gamma} \hat{v} \cdot \ell}{4}}^{\ell}<\infty\right]\right),
$$

where we used the inequality $X_{n} \cdot \ell \leq\left(X_{n} \cdot \hat{v}\right) \hat{v} \cdot \ell+\left|\Pi_{\hat{v}}\left(X_{n}\right)\right|_{2}$ to obtain the rightmost term in the last line of (5.12). The claim follows now from Proposition 4.5 and condition $(\mathbf{T})_{\ell}$.

The general procedure is now to consider columns, constructed by joining together boxes in direction $\hat{v}$. One then gathers columns to form tubes. 
We next make precise the terms "column" and "tube" by some further definitions. For $M>0$ and $M_{0}$ as above (the relation between $M$ and $M_{0}$ will appear in (5.21)), we attach to each $z \in M_{0} \mathbb{Z}^{d}$, the column

$$
\begin{array}{r}
\operatorname{Col}(z)=\left\{z^{\prime} \in M_{0} \mathbb{Z}^{d}: \exists j \in[0, J], z^{\prime}=z+j M_{0} e_{1}\right\}, \text { where } \\
J \text { is the smallest integer such that } J M_{0} \hat{v} \cdot \frac{l}{|l|_{2}} \geq 3 M .
\end{array}
$$

We choose $M_{1}>0$ an integer multiple of $M_{0}$ and define the tube attached to $z \in M_{0} \mathbb{Z}^{d}$ by:

$$
\begin{gathered}
\operatorname{Tube}(z)= \\
\left\{z^{\prime} \in M_{0} \mathbb{Z}^{d}: \exists j_{1}, j_{2} \ldots, j_{d} \in\left[0, \frac{M_{1}}{M_{0}}\right], z^{\prime}=z+\sum_{i=2}^{d} j_{i} M_{0} e_{i}\right\} .
\end{gathered}
$$

We stress that the key idea behind these definitions is the following strategy: one way for the walk to escape from slab $U_{M}$ is to move to one of the bottom blocks in Tube(0) of an appropriate column containing the greatest amount of good blocks and then move along this column up to its top. Under the choices that we will do later on, we will ensure that the walk escapes from $U_{M}$ by the boundary side $\partial^{+} U_{M}$, see Figure 2 . It will be convenient to introduce for $z \in M_{0} \mathbb{Z}^{d}$, the top of a tube as:

$$
\operatorname{Top}(z)=\bigcup_{z^{\prime} \in \operatorname{Tube}(z)} \partial^{+} \tilde{B}_{2}\left(z^{\prime}+J M_{0} e_{1}\right),
$$

along with the neighborhood of a tube as:

$$
\begin{gathered}
V(z)= \\
\left\{x \in \mathbb{Z}^{d}: \exists y \in \bigcup_{\substack{z^{\prime} \in \text { Tube(z) } \\
0 \leq j \leq J}} \tilde{B}_{1}\left(z^{\prime}+j M_{0} e_{1}\right),|x-y|_{1} \leq 3 d M_{1}\right\} .
\end{gathered}
$$

We need a lower bound on the $P_{0, \omega}$-probability for the event of reaching the top of a tube attached to the site $0 \in \mathbb{Z}^{d}$, before the walk exits from $V(0)$. To this end, we introduce the random minimum number of $M_{0}$-bad boxes contained in a column among columns in a tube as:

$$
n(z, \omega)=\min _{z^{\prime} \in \text { Tube }(z)}\left\{\sum_{j=0}^{J} \mathbb{1}_{\left\{z^{\prime}+M_{0} j e_{1} \text { is } M_{0}-\text { bad }\right\}}\right\} .
$$

imsart-aop ver. 2014/10/16 file: trenormrev.tex date: November 19, 2018 


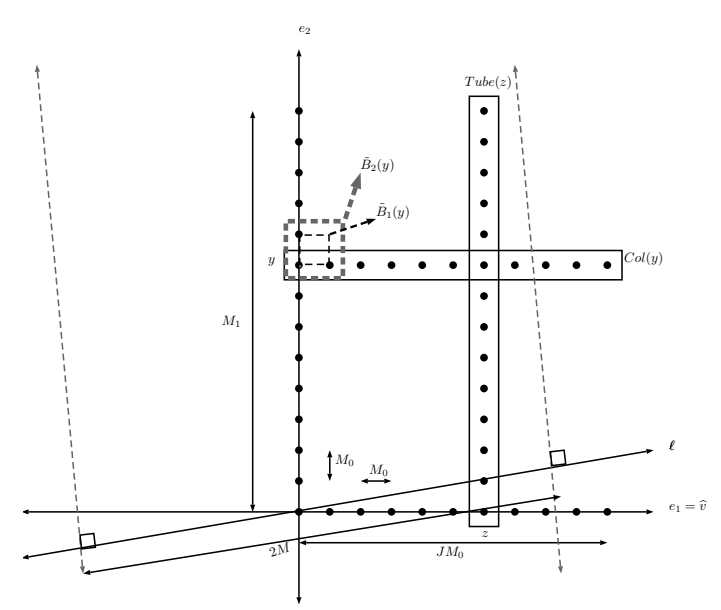

FIG 2. Schematic representation of definitions (5.13), (5.14) and strategy to escape of $U_{M}$ by $\partial^{+} U_{M}$. Dashed lines delimitate set $U_{M}$ and if $y$ is a point in Tube(0) with minimal amount of bad boxes on its column, the strategy is get to y from 0 and then use column on $y$ to escape by $\partial^{+} U_{M}$. The name columns comes from rotate counter clockwise this figure by a $\pi / 2$ angle.

Recalling our choice of $\kappa$ provided in (1.1), the proof of Lemma 3.3 in [Sz00] allows us to establish the next

Lemma 5.4. There exists $c_{10}>0$ such that for any $z \in M_{0} \mathbb{Z}^{d}$ and any

$$
x \in \bigcup_{\substack{z^{\prime} \in \text { Tube }(z), 0 \leq j \leq J}} \tilde{B}_{1}\left(z^{\prime}+j M_{0} e_{1}\right):=D(z),
$$

one has

$$
P_{x, \omega}\left[H_{T o p(z)}<T_{V(z)}\right] \geq(2 \kappa)^{c_{14}\left(M_{1}+J M_{0}^{\gamma}+n(z, \omega) M_{0}\right)}\left(\frac{1}{2}\right)^{J+1} .
$$

In virtue of Lemma 5.3 , we now choose $\gamma \in(5 / 9,1)$, such that:

$$
\chi:=\frac{1-\beta}{1-\gamma}<\beta<1
$$

and notice that such a choice is possible in view of assumption (5.6). We then choose

$$
\nu>1-\gamma
$$


and introduce for large $M$ :

$$
M_{0}=\rho_{1} M^{\chi}, M_{1}=\left[\rho_{2} M^{\beta-\chi}\right] M_{0}, N_{0}=\left[\rho_{3} M^{\beta-\chi}\right] \text {, }
$$

where the constants $\rho_{1}, \rho_{2}, \rho_{3}$, possibly depend on $\kappa,|l|_{2},|\hat{v}|_{2}, d, r, \delta$ and $c$ (cf.(5.4)). They are chosen so that for large $M$, the following requirements:

$$
\begin{aligned}
& \min \left\{(2 \kappa)^{c_{10} J M_{0}^{\gamma}},(2 \kappa)^{c_{10} M_{1}},(2 \kappa)^{c_{10} N_{0} M_{0}},\left(\frac{1}{2}\right)^{J+1}\right\} \\
& >\exp \left(-\frac{c}{5} M^{\beta}\right), \\
& \frac{N_{0}}{3}>(J+1) \frac{\left(e^{2}-1\right)}{M_{0}^{\nu}}, \text { and }
\end{aligned}
$$

any nearest neighbor path within $V(0)$, between 0 and Top $(0)$, first exits $U_{M}$ through $\partial^{+} U_{M}$.

are satisfied.

To see that such a choice is possible observe that it suffices to take $\rho_{1}$ large enough and $\rho_{2}=\rho_{3}=c\left(10 \rho_{1} c_{10} \log (1 /(2 \kappa))\right)^{-1}$, then (5.22) and (5.24) are satisfied for large $M$. As for (5.23), when $\beta<1$, it follows from the equality: $\beta-\chi=1-(1+\nu) \chi$.

Note that as a remark, the sites over which the environment events $\{z:$ is $M_{0}$-good $\}$ depend, where $z$ runs over the collection $\left(k_{1} M_{0}, \ldots, k_{d} M_{0}\right)$, with $k_{i}, i \in[1, d]$ non-negative integers, and $k_{1}+\ldots+k_{d}$ has a fixed parity; they are at least a $|\cdot|_{1}$-distance of $M_{0}-2 M_{0}^{\gamma}$ separated. Keeping this in mind, an application of Bunyakovsky-Cauchy-Schwarz inequality gives

$$
\begin{aligned}
\mathbb{P}\left[n(0, \omega)>N_{0}\right] & =\mathbb{P}\left[\bigcap _ { z ^ { \prime } \in \text { Tube } ( 0 ) } \left\{\sum_{j=0}^{J} \mathbb{1}_{\left.\left.\left\{z^{\prime}+M_{0} j e_{1} \text { is } M_{0} \text {-bad }\right\}>N_{0}\right\}\right]}\left[\bigcap_{\substack{z^{\prime} \in \text { Tube }(0) \\
k_{2}+\ldots+k_{d} \text { even }}}\left\{\sum_{j=0}^{J} \mathbb{1}_{\left\{z^{\prime}+M_{0} j e_{1} \text { is } M_{0} \text {-bad }\right\}}>N_{0}\right\}\right]^{\frac{1}{2}}\right.\right. \\
& \times \mathbb{P}\left[\bigcap_{\begin{array}{c}
z^{\prime} \in \text { Tube }(0) \\
k_{2}+\ldots+k_{d} \text { odd }
\end{array}}\left\{\sum_{j=0}^{J} \mathbb{1}_{\left\{z^{\prime}+M_{0} j e_{1} \text { is } M_{0}-\text { bad }\right\}}>N_{0}\right\}\right]^{\frac{1}{2}} .
\end{aligned}
$$


Using the previous remark for the last two expressions on the rightmost hand of (5.25) along successive conditioning to apply the mixing conditions (1.2) or (1.3), one gets:

$$
\leq \exp (C a)\left(\sup _{z^{\prime} \in \text { Tube }(0)} \mathbb{P}\left[\sum_{j=0}^{J} \mathbb{1}_{\left\{z^{\prime}+M_{0} j e_{1} \text { is } M_{0} \text {-bad }\right\}}>N_{0}\right]\right)^{\left[\frac{M_{1}}{2 M_{0}}\right]^{d-1}} .
$$

with the notation:

$$
a=\sum_{x \in H, y \in T} e^{-g|x-y|_{1}},
$$

where in turn for the mixing condition $(\mathbf{S M})_{C, g}(\mathrm{cf} .(1.2)), H, T$ denote the sets:

$$
\begin{aligned}
& H=\partial^{r}\left\{y \in \mathbb{Z}^{d}: y \in \tilde{B}_{2}(z), \text { for some } z \in \tilde{B}_{1}\left(M_{0} j e_{1}+\Sigma_{2 \leq i \leq d} k_{i} e_{i}\right),\right. \\
& \left.k_{i} \in\left[0, \frac{M_{1}}{M_{0}}\right],\left(k_{2}+\ldots+k_{d}\right)-(d-1) \frac{M_{1}}{M_{2}}=1(\bmod 2), j \in[0, J]\right\} \\
& T=\partial^{r}\left\{x \in \mathbb{Z}^{d}: x \in \tilde{B}_{2}(z), \text { for some } z \in \tilde{B}_{1}\left(M_{0} j e_{1}+\Sigma_{2 \leq i \leq d} \frac{M_{1}}{M_{0}} e_{i}\right),\right. \\
& j \in[0, J]\},
\end{aligned}
$$

and for the mixing condition $(\mathbf{S M G})_{C, g}($ cf. 1.3), the sets $H$ and $T$ will be switched to

$$
\begin{aligned}
& H=\left\{y \in \mathbb{Z}^{d}: y \in \tilde{B}_{2}(z), \text { for some } z \in \tilde{B}_{1}\left(M_{0} j e_{1}+\Sigma_{2 \leq i \leq d} k_{i} e_{i}\right),\right. \\
& \left.k_{i} \in\left[0, \frac{M_{1}}{M_{0}}\right],\left(k_{2}+\ldots+k_{d}\right)-(d-1) \frac{M_{1}}{M_{2}}=1(\bmod 2), j \in[0, J]\right\} \\
& T=\left\{x \in \mathbb{Z}^{d}: x \in \tilde{B}_{2}(z), \text { for some } z \in \tilde{B}_{1}\left(M_{0} j e_{1}+\Sigma_{2 \leq i \leq d} \frac{M_{1}}{M_{0}} e_{i}\right),\right. \\
& j \in[0, J]\} .
\end{aligned}
$$

By means of a similar argument as the one of Lemma 3.2, one sees that for large $M_{0}$ :

$$
C a \leq \exp \left(-\frac{g M_{0}}{2}\right)
$$

imsart-aop ver. 2014/10/16 file: trenormrev.tex date: November 19, 2018 
and consequently

$$
\leq \exp \left(e^{-\frac{g M_{0}}{4}}\right)\left(\sup _{z^{\prime} \in \text { Tube }(0)} \mathbb{P}\left[\sum_{j=0}^{J} \mathbb{1}_{\left\{z^{\prime}+M_{0} j e_{1} \text { is } M_{0} \text {-bad }\right\}}>N_{0}\right]\right)^{\left[\frac{M_{1}}{2 M_{0}}\right]^{d-1}} .
$$

Let us now observe that arguing as in [Sz00], page 125, when $Z$ is a Bernoulli random variable taking values onto $\{0,1\}$, with success probability smaller than $M_{0}^{-\nu}$, then $E[\exp (2 Z)] \leq 1+\left(e^{2}-1\right) / M_{0}^{\nu}$. As a result, restricting $j$ to even or odd integers, we conclude from Chebyshev's inequality with the help of: Lemma 5.12, successive conditioning, the mixing conditions $(\mathbf{S M})_{C, g}$ or $(\mathbf{S M G})_{C, g}$ and the choice of $\nu$ in (5.20), for large $M$

$$
\begin{aligned}
& \sup _{z^{\prime} \in \text { Tube(0) }} \mathbb{P}\left[\sum_{j=0}^{J} \mathbb{1}_{\left\{z^{\prime}+M_{0} j e_{1} \text { is } M_{0}-\text { bad }\right\}}>N_{0}\right] \\
& \leq 2 \exp \left(e^{-\frac{g M_{0}}{4}}\right) \exp \left(-N_{0}\right)\left(1+\frac{e^{2}-1}{M_{0}^{\nu}}\right) \\
& \leq 4 \exp \left(-N_{0}+(J+1) \frac{e^{2}-1}{M_{0}^{\nu}}\right) \stackrel{(5.23)}{\leq} \frac{1}{2} \exp \left(-\frac{N_{0}}{2}\right),
\end{aligned}
$$

where we have assumed in turn that $M$ is large enough so that

$$
\exp \left(e^{-\frac{g M_{0}}{4}}\right) \leq 2
$$

Therefore, for large $M$

$$
\mathbb{P}\left[n(0, \omega)>N_{0}\right] \leq \exp \left(-\frac{N_{0}}{2}\left[\frac{M_{1}}{2 M_{0}}\right]^{d-1}\right) .
$$

On the other hand, we have that on the event $\left\{n(0, \omega) \leq N_{0}\right\}$ :

$$
P_{0, \omega}\left[X_{T_{U_{M}}} \cdot \frac{l}{|l|_{2}} \geq M\right] \stackrel{(5.24)}{\geq} P_{0, \omega}\left[H_{T o p(0)}<T_{V(0)}\right] \stackrel{(5.18)-(5.22)}{>} e^{-c M^{\beta}} .
$$

Thus, one gets

$$
\underset{M}{\limsup } M^{d(\beta-\chi)} \log \mathbb{P}\left[P_{0, \omega}\left[X_{T_{U_{M}}} \cdot \frac{l}{|l|_{2}} \geq M\right] \leq e^{-c M^{\beta}}\right]<0,
$$

and the estimate (5.4) follows by letting $\gamma$ vary according to (5.19). 
5.2. Proof of Theorem 1.3. We now proceed with the proof of Theorem 1.3. The rough plan is to bound tails of the time $\tau_{1}$ and then we will apply Theorem 2 of [CZ02]. We begin with applying the previous atypical quenched estimate to obtain controls on the tails of the approximate regeneration times $\tau_{1}^{(L)}$. The precise statement will be the content of the following:

Proposition 5.5. There exist constants $c_{11}, c_{12}>0$ and $L_{0} \in|l|_{1} \mathbb{N}$, so that for each $L \in|l|_{1} \mathbb{N}$ with $L \geq L_{0}$ and for all $\alpha<1+\frac{4(d-1)}{13 d+4}$ :

$$
P_{0}\left[\tau_{1}^{(L)}>u\right] \leq e^{-c_{1} \kappa^{L}(\log (u))^{\alpha}}+e^{-c_{2}(\log (u))^{\alpha}} .
$$

Proof. We pick an $\alpha \in\left(1,1+\frac{4(d-1)}{13 d+4}\right)$ and consider for large $u$, the following choice of scales:

$$
\begin{aligned}
& \Delta(u)=\frac{1}{10 \sqrt{d}} \frac{\log (u)}{\log \left(\frac{1}{\kappa}\right)} \text { and } M(u)=N(u) \Delta(u), \\
& \text { where } N(u)=\left[(\log (u))^{\alpha-1}\right] .
\end{aligned}
$$

To simplify notation we drop the dependence on $u$ for the remainder of the proof.

In virtue of Lemma 5.1, the claim will follow once we can prove that:

$$
\underset{u}{\limsup } \log (u)^{-\alpha} \log \left(P_{0}\left[T_{C_{M}}>u\right]\right)<0 .
$$

Observe that for large $u$, one has (recall (5.1))

$$
\begin{array}{r}
P_{0}\left[T_{C_{M}}>u\right] \leq \mathbb{E}\left[\forall x \in C_{M}, P_{x, \omega}\left[T_{C_{M}} \leq \frac{u}{\log (u)^{\alpha}}\right] \geq \frac{1}{2}, P_{0, \omega}\left[T_{C_{M}}>u\right]\right] \\
+\mathbb{P}\left[\exists x_{1} \in C_{M}, P_{x_{1}, \omega}\left[T_{C_{M}}>\frac{u}{\log (u)^{\alpha}}\right]>\frac{1}{2}\right] .
\end{array}
$$

As a result of applying the Markov property, we see that

$$
\begin{aligned}
& \mathbb{E}\left[\forall x \in C_{M}, P_{x, \omega}\left[T_{C_{M}} \leq \frac{u}{\log (u)^{\alpha}}\right] \geq \frac{1}{2}, P_{0, \omega}\left[T_{C_{M}}>u\right]\right] \\
& \leq\left(\frac{1}{2}\right)^{\left[\log (u)^{\alpha}\right]} .
\end{aligned}
$$

Therefore, in order to prove (5.30), we need to obtain an upper bound as above for the second term on the right-hand side of (5.33). To this end, 
notice that when $x_{1}$ is such that $P_{x_{1}, \omega}\left[T_{C_{M}}>\frac{u}{\log (u)^{\alpha}}\right]>1 / 2$,

$$
\frac{1}{2} \frac{u}{\log (u)^{\alpha}} \leq E_{x_{1}, \omega}\left[T_{C_{M}}\right]=\sum_{x \in C_{M}} \frac{P_{x_{1}, \omega}\left[H_{x}<T_{C_{M}}\right]}{P_{x, \omega}\left[\widetilde{H}_{x}>T_{C_{M}}\right]}
$$

where $\widetilde{H}_{x}:=\inf \left\{n \geq 1: X_{n}=x\right\}, H_{x}:=H_{\{x\}}$ (cf. (2.2)). To see how the last equality above is obtained, we calculate

$$
\begin{gathered}
E_{x_{1}, \omega}\left[T_{C_{M}}\right]=\sum_{n \geq 0} P_{x_{1}, \omega}\left[T_{C_{M}}>n\right]=\sum_{x \in C_{M}}\left(\sum_{n \geq 0} P_{x_{1}, \omega}\left[T_{C_{M}}>n, X_{n}=x\right]\right) \\
\sum_{x \in C_{M}} E_{x_{1}, \omega}\left[\sum_{j=0}^{T_{C_{M}}} \mathbb{1}_{\left\{X_{j}=x\right\}}\right]=\sum_{x \in C_{M}} E_{x_{1}, \omega}\left[\sum_{j \geq 1} \mathbb{1}_{\left\{\left(H_{x}\right)_{j}<T_{C_{M}}\right\}}\right],
\end{gathered}
$$

where we have defined $\left(H_{x}\right)_{1}=H_{x}$ for $x \in C_{M}$, and then by recursion for $j>1$ :

$$
\left(H_{x}\right)_{j}=\widetilde{H}_{x} \circ \theta_{\left(H_{x}\right)_{j-1}}+\left(H_{x}\right)_{j-1} .
$$

Applying the strong Markov property to the last term in (5.36), we get

$$
\begin{aligned}
E_{x_{1}, \omega}\left[T_{C_{M}}\right] & =\sum_{x \in C_{M}} P_{x_{1}, \omega}\left[H_{x}<T_{C_{M}}\right] \sum_{j \geq 1} P_{x, \omega}\left[\widetilde{H}_{x}<T_{C_{M}}\right]^{j-1} \\
& =\sum_{x \in C_{M}} \frac{P_{x_{1}, \omega}\left[H_{x}<T_{C_{M}}\right]}{P_{x, \omega}\left[\widetilde{H}_{x}>T_{C_{M}}\right]} .
\end{aligned}
$$

Thus, coming back to (5.35), one has that there exists some $x_{2} \in C_{M}$ so that $\mathbb{P}$-a.s.

$$
P_{x_{2}, \omega}\left[\widetilde{H}_{x_{2}}>T_{C_{M}}\right] \leq\left|C_{M}\right| \frac{2(\log (u))^{\alpha}}{u}
$$

holds, on the event in the second term on the rightmost side of (5.33). Furthermore, notice that when $\omega \in \Omega$ is arbitrary, for $y:=x_{2} \in C_{M}$ as in (5.37), and $x \in \mathbb{Z}^{d}$ a nearest neighbour lattice point to $y+\ell K$ with $0<K \leq\left[\frac{1}{3} \frac{\log u}{\log \left(\frac{1}{2 \kappa}\right)}\right]$, by the elliptic assumption (1.1) we have that

$$
P_{y, \omega}\left[\widetilde{H}_{y}>T_{C_{M}}\right] \geq u^{-\frac{1}{3}} P_{x, \omega}\left[H_{y}>T_{C_{M}}\right] .
$$

imsart-aop ver. 2014/10/16 file: trenormrev.tex date: November 19, 2018 
Consequently for large $u$ we have $x \in C_{M}$ and

$$
P_{x, \omega}\left[H_{y}>T_{C_{M}}\right] \leq \frac{1}{\sqrt{u}}
$$

Thus, introducing the set

$$
V_{i}=\partial\left\{y \in \mathbb{Z}^{d}: y \cdot \frac{l}{|l|_{2}}<i \Delta\right\}, \text { for } i \in \mathbb{Z}^{d},
$$

we have that on the event

$$
\mathcal{E}=\bigcup_{x_{1} \in C_{M}}\left\{\omega \in \Omega: P_{x_{1}, \omega}\left[T_{C_{M}}>\frac{u}{(\log (u))^{\alpha}}\right]>\frac{1}{2}\right\}
$$

one can find $i_{0} \in[-N+1, N]$ and $x \in C_{M} \cap V_{i_{0}}$, such that

$$
P_{x, \omega}\left[\widetilde{T}_{\left(i_{0}-1\right) \Delta}^{l}>T_{C_{M}}\right] \leq \frac{1}{\sqrt{u}} .
$$

Let us remark that in order to obtain (5.41), we have used (5.38), the inequality: $2 \Delta+d \leq\left[\frac{1}{3} \frac{\log u}{\log \left(\frac{1}{2 \kappa}\right)}\right]$ and the fact that for any $y \in \mathbb{Z}^{d}$ a closest point to $x-(2 \Delta) \ell$, one has that

$$
P_{x, \omega}\left[\widetilde{T}_{\left(i_{0}-1\right) \Delta}^{l}>T_{C_{M}}\right] \leq P_{x, \omega}\left[H_{y}>T_{C_{M}}\right] .
$$

It will be convenient to introduce for $i \in \mathbb{Z}^{d}$ the random variables

$$
\mathfrak{X}_{i}:=\left\{\begin{array}{lll}
-\log \left(\inf _{x \in C_{M} \cap V_{i}} P_{x, \omega}\left[\widetilde{T}_{(i-1) \Delta}^{l}>T_{(i+1) \Delta}^{l}\right]\right) & \text { if } & C_{M} \cap V_{i} \neq \emptyset, \\
0 & \text { if } & C_{M} \cap V_{i}=\emptyset .
\end{array}\right.
$$

The next inequality is a consequence of induction along the strong Markov property (cf. [Sz00], pp 128). For $i \in[-N+1, N]$ and $x \in V_{i}$,

$$
P_{x, \omega}\left[\widetilde{T}_{(i-1) \Delta}^{l}>T_{C_{M}}\right] \geq \exp \left(-\sum_{j=i}^{N} \mathfrak{X}_{i}\right) .
$$

As a result from this last inequality and (5.41),

$$
\mathbb{P}[\mathcal{E}] \leq 2 N \sup _{i \in[-N+1, N]} \mathbb{P}\left[\mathfrak{X}_{i} \geq \frac{\log u}{2 N}\right]
$$


Note that for $i \in \mathbb{Z}$, and $\nu>0$ one has

$$
\mathbb{P}\left[\mathfrak{X}_{i}>\nu\right] \leq\left|C_{M}\right| \mathbb{P}\left[P_{0, \omega}\left[X_{T_{U_{\Delta}}} \cdot \frac{l}{|l|_{2}} \geq \Delta\right] \leq e^{-\nu}\right] .
$$

Therefore, using our version of an atypical quenched estimate given in (5.2), we get that whenever

$$
\left.1>2-\alpha \geq \frac{9 d+4}{13 d} \text { (and thus } \alpha \leq \frac{17 d-4}{13 d}\right)
$$

one has

$$
\mathbb{P}[\mathcal{E}] \leq \exp \left(-\widetilde{c}(\log u)^{\chi}\right)
$$

for all $\chi<d\left(\frac{13}{4}(2-\alpha)-\frac{9}{4}\right)$ and a suitable constant $\widetilde{c}=\widetilde{c}(d, \kappa, l)$.

In turn, the rightmost term in (5.44) is less than

$$
e^{-\widehat{c}(\log u)^{\alpha}}
$$

for a positive constant $\widehat{c}$, whenever $\alpha<\frac{17 d}{13 d+4} \leq \frac{17 d-4}{13 d}$. The proof is now complete from this last argument as was mentioned after (5.34).

We are ready to finish the proof of our main result.

Proof of Theorem 1.3. We observe that Proposition 5.5, via layer cake decomposition (cf. [Ru87], Chapter 8, Theorem 8.16) implies that there exists a deterministic constant $M=M(L)$, such that

$$
\mathbb{P}\left[\frac{\bar{E}_{0}\left[\left(\kappa^{L} \tau_{1}\right)^{3}, D^{\prime}=\infty \mid \mathfrak{F}_{0, L}\right]}{\bar{P}_{0}\left[D^{\prime}=\infty \mid \mathfrak{F}_{0, L}\right]}>M\right]=0 .
$$

The result of Theorem 1.3 follows from the central limit theorem of [CZ02].

6. On Kalikow's Condition. We will introduce in this section Kalikow's condition. We then prove that for a given $\ell \in \mathbb{S}^{d-1}$ the transient $(\mathbf{T})_{\ell}$ condition is satisfied whenever Kalikow's condition holds in the same direction. In the last part of this section, we will derive a ballistic strong law of large numbers, which is a slight extension of the main theorem in [RA03]. 
6.1. (T) is weaker than Kalikow's condition.

DeFinition 6.1. Kalikow's chain $\left(X_{n}\right)_{n \geq 0}$ on a connected $V \subsetneq \mathbb{Z}^{d}$ with $0 \in V$ is the canonical Markov chain with state space in $V \cup \partial V$, with transition probabilities given by

$$
\widehat{P}_{V}(x, x+e):= \begin{cases}\frac{E_{0}\left[\sum_{n=0}^{T_{V^{c}}} \mathbb{1}_{\left\{X_{n}=x\right\}} \omega(x, e)\right]}{E_{0}\left[\sum_{n=0}^{T_{V} c} \mathbb{1}_{\left\{X_{n}=x\right\}}\right]} & \text { for } \quad x \in V \text { and }|e|=1 \\ 1 & \text { for } \quad x \in \partial V \text { and } e=0 .\end{cases}
$$

For $x \in V \cup \partial V$ we will denote by $\hat{P}_{x, V}$ and $\hat{E}_{x, V}$ the law and expectation respectively of the corresponding Kalikow's chain starting from $x$ with transition probabilities as above. Setting the local drift $\hat{d}_{V}(x)=\hat{E}_{x, V}\left[X_{1}-X_{0}\right]$ at site $x$ of this walk, we say that Kalikow's condition is satisfied in direction $l \in \mathbb{R}^{d} \backslash\{0\}$ and we denote this by $(\boldsymbol{K})_{l}$ if there exists a constant $\delta(l)>0$ such that

$$
\inf _{x \in V, V} \hat{d}_{V}(x) \cdot l>\delta
$$

where the infimum runs over all the connected strict subsets $V$ of $\mathbb{Z}^{d}$, with $0 \in V$.

We quote here the following result owed to S. Kalikow [Ka81], which to some extend depicts the best known property of Kalikow's chain.

Suppose that $\hat{P}_{0, V}-$ a.s., $T_{V}$ is finite, then $P_{0}-$ a.s. $T_{V}$ is also finite, and $X_{T_{V}}$ has the same law under both $\hat{P}_{0, V}$ and $P_{0}$.

This property will be called as Kalikow's Proposition (see [Ka81], Proposition 1 for a proof).

Notice that when $|l|_{2}=1$, a straightforward application of CauchySchwarz inequality makes us see that the infimum in (6.1) is at most equal to 1. In [CZ01] was assumed at the nestling example of Section 5 that this infimum is close to 1 for $l=e_{1}$, besides a conditional version of Kalikow's condition. We will not need these further assumptions here.

Let us note that for $n \geq 0$,

$$
\mathfrak{M}_{n}^{V}:=X_{n}-X_{0}-\sum_{0 \leq j \leq n-1} \hat{d}_{V}\left(X_{j}\right)
$$

imsart-aop ver. 2014/10/16 file: trenormrev.tex date: November 19, 2018 
is a martingale for the canonical filtration of Kalikow's chain $\left(X_{n}\right)_{n \geq 0}$ starting from $x \in V \cup \partial V$, with state space in $V \cup \partial V$, where $V$ is a strict connected subset of $\mathbb{Z}^{d}$ with $0 \in V$. These martingales have increments bounded in Euclidean norm by 2, then Azuma-Hoeffding inequality (see [ASE92], page 85) turns out that

$$
\hat{P}_{x, V}\left[\mathfrak{M}_{n}^{V} \cdot w>A\right] \leq \exp \left(\frac{-A^{2}}{8 n}\right) \text { for } A>0, n \geq 0,|w|_{2}=1 .
$$

We recall that under Kalikow's condition the process $\left(H_{n}\right)_{n \in \mathbb{N}}$, defined by (see [Sz00], pp 101-103 for a proof):

$$
H_{n}:=\exp \left(-\eta X_{n} \cdot l\right)
$$

for all $\eta \in\left[0, \eta_{0}\right]$, where $\eta_{0}>0$ depends on $\delta$,

is a supermartingale under $\hat{P}_{x, V}$, for all strict connected subset $V$ of $\mathbb{Z}^{d}$ and $x \in V \cup \partial V$.

Letting $\ell \in \mathbb{S}^{d-1}$, the main result of this subsection comes in the next

Proposition 6.2. Assume $(\boldsymbol{K})_{\ell}$, then $(\boldsymbol{T})_{\ell}$ holds.

Proof. Assume condition $(\mathbf{K})_{\ell}$ and take $\delta>0$ as in the definition (6.1). In virtue of item $i$ ii) of Lemma 2.2 , we set $\mathrm{r}=2 / \delta$ and for large $M$ we estimate (cf. (2.1) for notation):

$$
P_{0}\left[X_{T_{B_{M, \mathrm{r} M, \ell}(0)}} \notin \partial^{+} B_{M, \mathrm{r} M, \ell}(0)\right],
$$

where as usual the underlying rotation $R$ entering in the definition of the box $B_{M, \mathrm{r} M, \ell}(0)$ satisfies $R\left(e_{1}\right)=\ell$.

Notice that denoting $B_{M}$ the box $B_{M, \mathrm{r} M, \ell}(0)$, one has

$$
\begin{aligned}
& P_{0}\left[X_{T_{B_{M}, \mathrm{r} M, \ell}(0)} \notin \partial^{+} B_{M, \mathrm{r} M, \ell}(0)\right] \\
& \stackrel{(6.2)}{=} \hat{P}_{0, B_{M}}\left[X_{T_{B_{M}}} \cdot \ell<M\right] \leq \hat{P}_{0, B_{M}}\left[T_{B_{M}}>\mathrm{r} M\right] \\
& +\hat{P}_{0, B_{M}}\left[T_{B_{M}} \leq \mathrm{r} M, X_{T_{B_{M}}} \cdot \ell<M\right] .
\end{aligned}
$$

We set $N=[\mathrm{r} M]$ and observe that $\hat{P}_{0, B_{M}}$-a.s. on $T_{B_{M}}>\mathrm{r} M$,

$$
\mathfrak{M}_{N} \cdot \ell<-M / 2 .
$$

Hence, using Azuma-Hoeffding inequality (6.4), we find that

$$
\hat{P}_{0, B_{M}}\left[T_{B_{M}}>\mathrm{r} M\right] \leq \exp \left(-\frac{M^{2}}{16 N}\right) .
$$

imsart-aop ver. 2014/10/16 file: trenormrev.tex date: November 19, 2018 
On the other hand, applying Chevyshev's inequality and the optional stopping theorem along the supermartingale in (6.5), we get

$$
\begin{aligned}
\hat{P}_{0, B_{M}}\left[T_{B_{M}} \leq \mathrm{r} M, X_{T_{B_{M}}} \cdot \ell<M\right] & =\hat{P}_{0, B_{M}}\left[X_{T_{B_{M}}} \cdot \ell \leq-M\right] \\
& \leq \exp (-\eta M) .
\end{aligned}
$$

Inserting (6.7) and (6.8) into (6.6) we complete the proof.

The class of random environments studied in the present article extends the i.i.d. class. Alongside our ballisticity condition $(\mathbf{T})_{\ell}$ extends the previous i.i.d. condition $\left.(T)\right|_{\ell}$ as well (cf. Theorem 1.1 of [Sz02]). Recently in the framework of i.i.d. random environments, we have been able to prove the equivalence $\left.\left.(T)\right|_{\ell} \leftrightarrow\left(T^{\prime}\right)\right|_{\ell}$ (cf. Theorem 2.1 of [GR18]). On the other hand, Sznitman in [Sz03] has constructed ballistic walk examples satisfying $\left.\left(T^{\prime}\right)\right|_{\ell}$ where Kalikow's condition breaks down, for all dimension $d \geq 3$. Thus, at least for dimensions $d \geq 3$, condition $(\mathbf{T})_{\ell}$ is strictly weaker than $(\mathbf{K})_{\ell}$.

6.2. Ballistic Regime under Kalikow's condition. The next result can be thought as an alternative proof of the law of large numbers in [RA03] under Kalikow's condition, however a slightly more general mixing condition will be considered. Precisely one has:

Theorem 6.3. Let $C, g>0$. Assume that the RWRE fulfils conditions $(\boldsymbol{K})_{l}$ and either $(\boldsymbol{S} \boldsymbol{M} \boldsymbol{G})_{C, g}$ or $(\boldsymbol{S M})_{C, g}$, then there exists a deterministic vector $v \in \mathbb{R}^{d} \backslash\{0\}$, so that $P_{0}$-a.s.

$$
\lim _{n \rightarrow \infty} \frac{X_{n}}{n}=v
$$

with $v \cdot l>0$.

Let us begin by recalling the following (cf. [Gu14], Lemma 9 for a proof)

Lemma 6.4. Let $a \in(0,1)$. Suppose that a sequence $\left(X_{n}\right)_{n \geq 1}$ of nonnegative random variables satisfies

$$
a \leq \frac{d P\left[X_{n+1} \in \cdot \mid X_{n}, \ldots, X_{1}\right]}{d \mu} \leq a^{-1}
$$

for all $n \geq 1$, where $P$ and $\mu$ are probability measures. Setting $m_{\mu}=$ $\int x d \mu(x)$, then $P$-a.s. one has that

$$
a m_{\mu} \leq \liminf _{n \rightarrow \infty} \sum_{k=1}^{n} X_{k} / n \leq \limsup _{n \rightarrow \infty} \sum_{k=1}^{n} X_{k} / n \leq a^{-1} m_{\mu} .
$$

imsart-aop ver. 2014/10/16 file: trenormrev.tex date: November 19, 2018 
The key result for our proof comes in the next proposition, where a limiting but possibly vanishing velocity is proven. For $l \in \mathbb{Z}^{d}$ we will always assume $(\mathbf{K})_{l}$ (this is not a restriction, see Subsection 2.2) and either: $(\mathbf{S M})_{C, g}$ or (SMG) $)_{C, g}$. As a result of Proposition 6.2 , for $L \in|l|_{1} \mathbb{N}$ we can construct the random variable $\tau_{1}^{(L)}$ along vector $l$.

Proposition 6.5. Assume $(\boldsymbol{K})_{l}$ an either: $(\boldsymbol{S} \boldsymbol{M})_{C, g}$ or $(\boldsymbol{S} \boldsymbol{M} \boldsymbol{G})_{C, g}$. Then there exists $v \in \mathbb{R}^{d}$ deterministic, such that $P_{0}$-a.s.

$$
\lim _{n \rightarrow \infty} \frac{X_{n}}{n} \rightarrow v
$$

Proof. We complete the unit vector $\frac{l}{|l|_{2}}$ to form an orthonormal base of $\mathbb{R}^{d}$, which we will denote by $\mathfrak{V}:=\left\{\frac{l}{|l|}, w_{2}, \ldots, w_{d-1}\right\}$. We need the following claim whose proof will be postponed:

For all vector $w \in \mathfrak{V}$, there exist $\widehat{C}>0$ and $L_{0} \in|l|_{1} \mathbb{N}$ so that for all $L \geq L_{0}$ one has that

$$
\limsup _{n \rightarrow \infty}\left|\kappa^{L} \frac{X_{\tau_{n}} \cdot w}{n}-\kappa^{L} \bar{E}_{0}\left[X_{\tau_{1}} \cdot w \mid D^{\prime}=\infty\right]\right| \leq e^{-\widehat{C} L}
$$

Assuming the previous claim we can now prove the proposition. Pick a nondecreasing sequence $\left(k_{n}\right)_{n \geq 0}$, such that

$$
\tau_{k_{n}} \leq n<\tau_{k_{n}+1}
$$

By the very definition of the renewal structure we have $\bar{P}_{0}$-a.s: $k_{n}$ goes to $\infty$ as $n \rightarrow \infty$. Furthermore, with the help of Corollary 3.3 we can use Lemma 6.4 to see that

$$
\limsup _{n \rightarrow \infty}\left|\kappa^{L} \frac{\tau_{n}}{n}-\kappa^{L} \bar{E}_{0}\left[\tau_{1} \mid D^{\prime}=\infty\right]\right| \leq e^{-\bar{C} L}
$$

and by the claim

$$
\limsup _{n \rightarrow \infty}\left|\kappa^{L} \frac{X_{\tau_{n}}}{n}-\kappa^{L} \bar{E}_{0}\left[X_{\tau_{1}} \mid D^{\prime}=\infty\right]\right|_{2} \leq e^{-\bar{C} L},
$$

for a suitable positive constant $\bar{C}$. Therefore, using the decomposition

$$
\frac{X_{n}}{n}=\frac{X_{\tau_{k_{n}}}}{k_{n}} \frac{k_{n}}{n}+\frac{X_{n}-X_{\tau_{k_{n}}}}{n},
$$

imsart-aop ver. 2014/10/16 file: trenormrev.tex date: November 19, 2018 
there exists a positive constant $C_{6}$, so that

$$
\limsup _{n \rightarrow \infty}\left|\frac{X_{n}}{n}-\frac{\bar{E}_{0}\left[X_{\tau_{1}} \mid D^{\prime}=\right]}{\bar{E}_{0}\left[\tau_{1} \mid D^{\prime}=\infty\right]}\right| \leq e^{-C_{6} L} .
$$

where we have used that

$$
\limsup _{n \rightarrow \infty}\left|\frac{X_{n}-X_{\tau_{k_{n}}}}{n}\right|=0
$$

which will be implied once we show that there exists $C_{7}>0$ such that

$$
\begin{aligned}
& \limsup _{n \rightarrow \infty}\left|\frac{\sum_{1 \leq j \leq n} \sup _{0 \leq i \leq \tau_{1}}\left|X_{i \circ \theta_{\tau_{j}}}-X_{\tau_{j}}\right|}{n}-\bar{E}_{0}\left[\sup _{0 \leq i \leq \tau_{1}}\left|X_{i}\right| \mid D^{\prime}=\infty\right]\right| \\
& \quad \leq e^{-C_{7} L} .
\end{aligned}
$$

In order to prove (6.11), we apply Lemma 6.4 together with Corollary 3.3 once again, to get

$$
\begin{aligned}
\limsup _{n \rightarrow \infty} \mid & \left|\frac{\sum_{1 \leq j \leq n} \sup _{0 \leq i \leq \tau_{1}}\left|X_{i \circ \theta_{\tau_{j}}}-X_{\tau_{j}}\right|}{n}-\bar{E}_{0}\left[\sup _{0 \leq i \leq \tau_{1}}\left|X_{i}\right| \mid D^{\prime}=\infty\right]\right| \\
& \leq 1-\exp \left(-2 e^{(-g L) / 4}\right)
\end{aligned}
$$

which implies the claim in (6.11). The proposition follows now by letting (recall our notation $\tau_{1}=\tau_{1}^{(L)}$ )

$$
v=\lim _{L \rightarrow \infty} \frac{\bar{E}_{0}\left[X_{\tau_{1}} \mid D^{\prime}=\infty\right]}{\bar{E}_{0}\left[\tau_{1} \mid D^{\prime}=\infty\right]},
$$

with the convention that $L$ in the limit runs over $\mathbb{N}|l|_{1}$. To see that such limit exists, notice

$$
\lim _{L \rightarrow \infty} \bar{E}_{0}\left[\tau_{1} \mid D^{\prime}=\infty\right]=\sup _{L \in \mathbb{N}|l|_{1}} \bar{E}_{0}\left[\tau_{1} \mid D^{\prime}=\infty\right] \in(0, \infty]
$$

Setting:

$$
\mathfrak{T}_{1}(L):=\kappa^{L} \bar{E}_{0}\left[\tau_{1} \mid D^{\prime}=\infty\right],
$$

by virtue of Proposition 4.4, we have two cases: $\lim _{L \rightarrow \infty} \mathfrak{T}_{1}(L)=\infty$ or there exists $k_{3} \in\left[k_{2}, \infty\right)$ such that $\lim _{L \rightarrow \infty} \mathfrak{T}_{1}(L)=k_{3}$. In the former case, using Corollary 4.3 we have that $v=0$. In the second case we define for integer $n>1$

$$
v_{n}:=\frac{\bar{E}_{0}\left[X_{\tau_{1}^{\left(n|l|_{1}\right)}} \mid D^{\prime}=\infty\right]}{\bar{E}_{0}\left[\tau_{1}^{\left(\left.n|l|\right|_{1}\right)} \mid D^{\prime}=\infty\right]} .
$$


From the very definition of the renewal structure we have that for large integers $m>n$

$$
\begin{aligned}
& \left|\bar{E}_{0}\left[X_{\tau_{1}^{\left(m|l| l_{1}\right)}}-X_{\tau_{1}^{\left(n \mid l l_{1}\right)}} \mid D^{\prime}=\infty\right]\right|_{2} \\
& \leq \bar{E}_{0}\left[\sup _{0 \leq i \leq \tau_{1}^{\left((m-n)|l|_{1}\right)}}\left|X_{n}\right|_{2} \mid D^{\prime}=\infty\right]
\end{aligned}
$$

and,

$$
\bar{E}_{0}\left[\tau_{1}^{\left(m|l|_{1}\right)}-\tau_{1}^{\left(n|l|_{1}\right)} \mid D^{\prime}=\infty\right] \leq \bar{E}_{0}\left[\tau_{1}^{\left((m-n)|l|_{1}\right)} \mid D^{\prime}=\infty\right] .
$$

Using both estimates (6.13)-(6.14) and Proposition 4.4, it is routine to prove that for large $m$ and $n$ with $m>n$,

$$
\left|v_{m}-v_{n}\right| \leq 2 \kappa^{n|l|_{1}}
$$

Therefore the limiting velocity in (6.12) exists.

We now turn to prove claim (6.10). Let $w \in \mathfrak{V}$ and set (with the notation $\left.\tau_{0}=0\right)$

$$
Z_{i}=\kappa^{L}\left(X_{\tau_{i}}-X_{\tau_{i-1}}\right) \cdot w
$$

for integer $i \geq 1$. Using a coupling decomposition argument (cf. [CZ01]), we can enlarge the probability space where the sequence $\left(Z_{i}\right)_{i \geq 1}$ is defined. We will still denote the new probability measure by $\bar{P}_{0}$ in order to support the following:

- There exist two i.i.d. sequences $\left(\tilde{Z}_{i}\right)_{i \geq 1}$ and $\left(\Delta_{i}\right)_{i \geq 1}$ such that $\tilde{Z}_{1}$ is distributed according to the distribution $\bar{P}_{0}\left[Z_{1} \in \cdot D^{\prime}=\infty\right]$, and $\Delta_{1}$ is Bernoulli distributed with values onto $\{0,1\}$ and success probability $\bar{P}_{0}\left[\Delta_{1}=1\right]=\exp (-\tilde{c} L)$, for some suitable and fixed constant $\tilde{c}>0$.

- There exists a third sequence $\left(W_{i}\right)_{i \geq 1}$ so that for $i \geq 1$ one has that $\Delta_{i}$ is independent of $W_{i}$ and the $\sigma$-algebra $\mathcal{G}_{i}$ defined by

$$
\mathcal{G}_{i}=\sigma\left(\left(Z_{j}\right)_{j \leq i-1},\left(\Delta_{j}\right)_{j \leq i-1}\right)
$$

with the convention that $\mathcal{G}_{1}$ is the trivial $\sigma$-algebra.

- In the new probability space, for integer $i \geq 1$ one has the decomposition:

$$
Z_{i}=\tilde{Z}_{i}\left(1-\Delta_{i}\right)+\Delta_{i} W_{i}
$$


Therefore, one has on that large probability space

$$
\frac{\kappa^{L} X_{\tau_{n}} \cdot w}{n}=\frac{\sum_{i=1}^{n} Z_{i}}{n}=\frac{\sum_{i=1}^{n} \tilde{Z}_{i}}{n}-\frac{\sum_{i=1}^{n} \tilde{Z}_{i} \Delta_{i}}{n}+\frac{\sum_{i=1}^{n} \Delta_{i} W_{i}}{n} .
$$

We are going now to estimate each one of the terms to the right of (6.15). The strong law of large numbers implies that $\bar{P}_{0}$-a.s.

$$
\frac{\sum_{i=1}^{n} \tilde{Z}_{i}}{n} \rightarrow \bar{E}_{0}\left[\tilde{Z}_{1}\right]=\bar{E}_{0}\left[\kappa^{L} X_{\tau_{1}} \cdot w \mid D^{\prime}=\infty\right]
$$

and together with Corollary $4.3, \bar{P}_{0}$-a.s. we have

$$
\begin{aligned}
& \frac{\sum_{i=1}^{n} \tilde{Z}_{i} \Delta_{i}}{n} \rightarrow \bar{E}_{0}\left[\tilde{Z}_{1} \Delta_{1}\right] \leq\left(\bar{E}_{0}\left[\left(\kappa^{L} X_{\tau_{1}} \cdot w\right)^{2} \mid D^{\prime}=\infty\right] \exp (-\tilde{c} L)\right)^{\frac{1}{2}} \\
& \leq \exp (-\mathfrak{c} L)
\end{aligned}
$$

for some positive constant $\mathfrak{c}$.

We next turn to bound from above the third expression on the right most side of 6.15. This will be performed following a close argument to the one of [CZ01], pp 894-895. Define $\bar{W}_{i}:=\bar{E}_{0}\left[W_{i} \mid \mathcal{G}_{i}\right]$ and $M_{n}=\sum_{i=1}^{n}\left(\Delta_{i}\left(W_{i}-\bar{W}_{i}\right)\right) / i$, for integers $i$ and $n$ greater than 0 . Notice that $M_{n}$ is a $\mathcal{G}_{n}$-martingale centered at 0. We apply Burkholder-Gundy maximal inequality (cf. [Wi91], Section 14.18) and Corollary 4.3 to get

$$
\bar{E}_{0}\left[\left|\sup _{n \geq 1} M_{n}\right|^{2}\right] \leq C_{8} \bar{E}_{0}\left[\sum_{i \geq 1} \frac{\left(\Delta_{i}\left(W_{i}-\bar{W}_{i}\right)\right)^{2}}{i^{2}}\right] \leq \widetilde{C}_{3}
$$

for some constants $C_{8}$ and $\widetilde{C}_{3}$. This implies that $M_{n}$ almost surely converges to an integrable random variable. Consequently, applying now Kronecker's lemma (cf. [Wi91], Section 12.7), one has that $\bar{P}_{0}$-a.s. $H_{n}:=\Sigma_{i=1}^{n}\left(\Delta_{i}\left(W_{i}-\right.\right.$ $\left.\left.\bar{W}_{i}\right)\right) / n \rightarrow 0$. Since $\Delta_{i}$ is independent of $\mathcal{G}_{i}$, using Corollary 4.3 and Jensen's inequality we get

$$
\begin{aligned}
\left|\bar{W}_{i}\right| & \leq\left(\bar{E}_{0}\left[\left|W_{i}\right|^{2} \mid \mathcal{G}_{i}\right]\right)^{\frac{1}{2}} \\
& \leq\left(\exp \left(e^{-(g L) / 4}\right) \bar{E}_{0}\left[\left(\kappa^{L} X_{\tau_{1}} \cdot w\right)^{2} \mid D^{\prime}=\infty\right]\right)^{\frac{1}{2}} \exp \left(\frac{\tilde{c} L}{2}\right) \\
& \leq \bar{C}_{4} \exp \left(\frac{\tilde{c} L}{2}\right)
\end{aligned}
$$

imsart-aop ver. 2014/10/16 file: trenormrev.tex date: November 19, 2018 
where $\bar{C}_{4}>0$ is a constant. Hence

$$
\begin{aligned}
\sum_{i=1}^{n} \frac{\Delta_{i} \bar{W}_{i}}{n} & \leq \bar{C}_{4} \exp \left(\frac{\tilde{c} L}{2}\right) \sum_{i=1}^{n} \frac{\Delta_{i}}{n} \\
& \stackrel{L L N}{\rightarrow} \bar{C}_{4} \exp \left(-\frac{\tilde{c} L}{2}\right) .
\end{aligned}
$$

Thus, combining (6.16), (6.17) and (6.18) we have proven claim (6.10).

We need another auxiliary result in order to prove that the limiting velocity $v$ is a non-vanishing one. Specifically, Kalikow's condition admits a ballistic characterization (cf. [SZ99], pp 1861-1862 for a proof):

Lemma 6.6. For any finite connected set $U$ containing 0,

$$
E_{0}\left[T_{U}\right] \leq \frac{1}{\delta} E_{0}\left[X_{T_{U}} \cdot l\right]
$$

where $\delta$ is as in (6.1) and $T_{U}$ is defined in (2.2).

We are now ready to prove Theorem 6.3.

Proof of Theorem 6.3. Fixing $L \geq L_{0}$ with $L \in|l|_{1} \mathbb{N}$, we consider for $m \geq 0$, the nondecreasing sequence $k_{m}^{\prime}, P_{0}$-almost surely tending to $\infty$ as $m$ does (where as before, we use the convention $\tau_{0}^{(L)}=0$ ), such that

$$
\tau_{k_{m}^{\prime}}^{(L)} \leq T_{m}^{l}<\tau_{k_{m}^{\prime}+1}^{(L)}
$$

From the definitions of the sequence $\left(\tau_{k}^{(L)}\right)_{k \geq 1}$ (and from now on, we drop the index $L$ for $\tau_{k}^{(L)}$ and $\left.X_{\tau_{k}}^{(L)}\right)$, one has that $\bar{P}_{0 \text {-a.s. }}$

$$
l \cdot X_{n}<l \cdot X_{\tau_{k}} \leq l \cdot X_{n^{\prime}} \text {, for } 0 \leq n<\tau_{k} \leq n^{\prime} .
$$

Hence, for $m \geq 0, \bar{P}_{0}$-a.s.

$$
X_{\tau_{k_{m}^{\prime}}} \cdot l \leq X_{T_{m}^{l}} \cdot l \leq X_{\tau_{k_{m}^{\prime}+1}} \cdot l
$$

and on the other hand, one has

$$
\left|X_{T_{m}} \cdot l-m\right|_{2} \leq \sup _{i \in[1, d]}\left|l_{i}\right| .
$$

Notice first that by Lemma 6.4 and Corollary 3.3 one has that $\bar{P}_{0 \text {-a.s. }}$

$$
\liminf _{m \rightarrow \infty} \frac{k_{m}^{\prime}}{X_{\tau_{k_{m}^{\prime}}} \cdot l} \geq \exp \left(-e^{-(g L) / 4}\right) \frac{1}{\bar{E}_{0}\left[X_{\tau_{1}} \cdot l \mid D^{\prime}=\infty\right]},
$$

imsart-aop ver. 2014/10/16 file: trenormrev.tex date: November 19, 2018 
together with

$$
\liminf _{m \rightarrow \infty} \frac{\tau_{k_{m}^{\prime}}}{k_{m}^{\prime}} \geq \exp \left(-e^{-(g L) / 4}\right) \bar{E}_{0}\left[\tau_{1} \mid D^{\prime}=\infty\right]
$$

Moreover, a similar argument to the one in (6.11) gives the following upper bound $\bar{P}_{0}$-a.s.

$$
\limsup _{m \rightarrow \infty} \frac{\left|\left(X_{\tau_{k_{m}^{\prime}+1}}-X_{\tau_{k_{m}^{\prime}}}\right) \cdot l\right|}{m}=0 .
$$

Hence, by the very definition of the sequence $k_{m}^{\prime}$, estimates (6.22), (6.23) and (6.24); we have $\bar{P}_{0}$-a.s.

$$
\begin{aligned}
\liminf _{m \rightarrow \infty} \frac{T_{m}^{l}}{m} & \geq \liminf _{m \rightarrow \infty} \frac{\tau_{k_{m}^{\prime}}}{m}=\frac{\tau_{k_{m}^{\prime}}}{k_{m}^{\prime}} \frac{k_{m}^{\prime}}{X_{\tau_{k_{m}^{\prime}}} \cdot l} \frac{X_{\tau_{k_{m}^{\prime}}} \cdot l}{m} \\
& \geq \liminf _{m \rightarrow \infty} \frac{\tau_{k_{m}^{\prime}}}{k_{m}^{\prime}} \liminf _{m \rightarrow \infty} \frac{k_{m}^{\prime}}{X_{\tau_{k_{m}^{\prime}}} \cdot l} \liminf _{m \rightarrow \infty} \frac{X_{\tau_{k_{m}^{\prime}}} \cdot l}{m} \\
& \geq\left(\exp \left(-e^{-(g L) / 4}\right) \bar{E}_{0}\left[\tau_{1} \mid D^{\prime}=\infty\right]\right)\left(\frac{\exp \left(-e^{-(g L) / 4}\right)}{\bar{E}_{0}\left[X_{\tau_{1}} \cdot l \mid D^{\prime}=\infty\right]}\right),
\end{aligned}
$$

where to obtain the rightmost estimate in (6.25), we have used:

$$
\liminf _{m \rightarrow \infty} \frac{X_{\tau_{k_{m}^{\prime}}} \cdot l}{m}=\liminf _{m \rightarrow \infty}\left(\frac{X_{T_{m}^{l}} \cdot l}{m}-\frac{\left(X_{T_{m}^{l}}-X_{\tau_{k_{m}^{\prime}}}\right) \cdot l}{m}\right)=1
$$

which is satisfied, by virtue of (6.21) and (6.24). Furthermore, by an exhaustion of $\left\{y \in \mathbb{Z}^{d}: y \cdot l<m\right\}$ by finite subsets of $\mathbb{Z}^{d}$, one sees that applying Lemma 6.19 and Fatou's Lemma

$$
\bar{E}_{0}\left[\liminf _{m \rightarrow \infty} \frac{T_{m}^{l}}{m}\right] \leq \liminf _{m \rightarrow \infty} \bar{E}_{0}\left[\frac{T_{m}^{l}}{m}\right] \leq \frac{1}{\delta} .
$$

Therefore, Kalikow's condition implies that there exists a constant $f=$ $f(g, d, l, \delta)$ which does not depend on $L$ so that

$$
\bar{E}_{0}\left[\kappa^{L} \tau_{1} \mid D^{\prime}=\infty\right] \leq f .
$$

As a result $v:=\lim _{L \rightarrow \infty} \bar{E}_{0}\left[X_{\tau_{1}} \mid D^{\prime}=\infty\right] / \bar{E}_{0}\left[\tau_{1} \mid D^{\prime}=\infty\right]$ is a non-vanishing limiting velocity and furthermore, there exists a constant $k_{4}>0$ such that $v \cdot l \geq k_{4}$ by Proposition 4.4 . 
Acknowledgments. I wish to thank Alejandro Ramírez my former $\mathrm{PhD}$. advisor for suggesting me this problem and some useful indications about the paper writing. This work was almost completely done when I held a postdoctoral position in the beautiful and amazing country of Brazil at the Universidade Federal do Rio de Janeiro. Therefore I thank Maria Eulalia Vares and Glauco Valle for the opportunity, suggestions, corrections and hearing me in weekly held seminars when I was developing this work. I am pretty sure that vocês estiverom torçendo para que eu conseguisse. An anonymous referee has contributed with a detailed report and advised me about several inaccuracies in a preliminary version, I am grateful for that. Last but not least, my best thanks to Alain-Sol Sznitman, who helped me to improve on the result of an earlier version in Proposition 5.5.

\section{References.}

[ASE92] Alon, N., Spencer, J., Erdös, P. The probabilistic method. New York: John Wiley and Sons (1992).

[BDR14] N. Berger, A. Drewitz and A.F. Ramírez. Effective Polynomial Ballisticity Conditions for Random Walk in Random Environment. Comm. Pure Appl. Math., 67, pp 19471973, (2014).

[Ch62] A. Chernov. Replication of a multicomponent chain by the lightning mechanism. Biophysics 12, pp 336-341, (1962).

[CZ01] F. Comets and O. Zeitouni. A law of large numbers for random walks in random mixing environments. Ann. Probab. 32 , no. 1B, pp 880-914, (2004).

[CZ02] F. Comets and O. Zeitouni. Gaussian fluctuations for random walks in random mixing environments. Probability in mathematics. Israel J. Math. 148, pp 87-113, (2005).

[D094] P. Doukhan. Mixing: Properties and Examples. Springer, New York, (1994).

[DS85] R. Dobrushin and S. Shlosman. Constructive criterion for the uniqueness of Gibbs fields. Statistical Physics and Dynamical Systems (J. Fritz, A. Jaffe and D. Szs eds.) Birkh/iuser, Basel, pp 347-370, (1985).

[DR14] A. Drewitz and A.F. Ramírez. Selected topics in random walks in random environment. Topics in percolative and disordered systems, pp 2383, Springer Proc. Math. Stat., 69, Springer, New York, (2014).

[GR15] E. Guerra and A. F. Ramírez. Almost exponential decay for the exit probability from slabs of ballistic RWRE. Electron. J. Probab. 20, paper no. 24, $17 \mathrm{pp}$, (2015).

[GR17] E. Guerra and A. F. Ramírez. Asymptotic direction for random walks in mixing random environments. Electron. J. Probab. 22, paper no. 92, 41 pp, (2017).

[GR18] E. Guerra and A. F. Ramírez. A proof of Sznitman's conjecture about ballistic RWRE. arXiv:1809.02011 [math.PR] (2018).

[Gu14] X. Guo. On the limiting velocity of random walks in mixing random environment Ann. Inst. H. Poincar Probab. Statist., 50 (2) , pp 375-402, (2014).

[IG80] M. Iosifescu and S. Grigorescu. Dependence with Complete Connections and its Applications. Cambridge University Press, (1990).

[Ka81] S. Kalikow. Generalized random walks in random environment. Ann. Probab. 9, pp 753-768, (1981). 
[Ke77] H. Kesten. A renewal theorem for random walk in a random environment. Proc. Sympos. Pure Math. 31, pp 6777, (1977).

[Ko85] S. Kozlov. The method of averaging and walks in inhomogeneous environments. Russian Math. Surveys 40, pp 73145, (1985).

[Ma99] F. Martinelli Lectures on Glauber dynamics for discrete spin systems., Lecture Notes in Mathematics 1717, Springer, Berlin, pp 96-181, (1999).

[RA03] F. Rassoul-Agha. The point of view of the particle on the law of large numbers for random walks in a mixing random environment. Ann. Probab. 31 , no. 3, pp 1441-1463, (2003).

[Ru87] W. Rudin. Real and Complex Analysis. McGRAW-HILL International Editions, (1987).

[Si82] Y. S. Sinai. The limiting behavior of a one-dimensional random walk in a random envrionment. Theory Prob. Appl. 27, no. 2, pp 247-258, (1982).

[Sz00] A.S. Sznitman. Slowdown estimates and central limit theorem for random walks in random environment. J. Eur. Math. Soc. 2, no. 2, pp 93-143, (2000).

[Sz01] A.S. Sznitman. On a class of transient random walks in random environment. Ann. Probab. 29 (2), pp 724-765, (2001).

[Sz02] A.S. Sznitman. An effective criterion for ballistic behavior of random walks in random environment. Probab. Theory Related Fields 122, no. 4, pp 509-544, (2002).

[Sz03] A.S, Sznitman. On new examples of ballistic random walks in random environment. Ann. Probab. 31, no. 1, pp 285-322, (2003).

[SZ99] A.S. Sznitman and M. Zerner. A law of large numbers for random walks in random environment. Ann. Probab. 27 , no. 4, pp 1851-1869, (1999)

[Wi91] D. Williams. Probability with Martingales. Cambridge Univ. Press, (1991).

[Ze04] O. Zeitouni. Random walks in random environment. Lectures on probability theory and statistics, pp 189312, Lecture Notes in Math., 1837, Springer, Berlin, (2004).

E-MAIL: eaguerra@mat.puc.cl 